\title{
Exponential Observers for Distributed Tubular (Bio)Reactors
}

\author{
Míriam R. García, Carlos Vilas, Julio R. Banga, \\ Antonio A. Alonso * \\ Process Engineering Group, IIM-CSIC, Eduardo Cabello 6, 36208 Vigo, Spain.
}

\begin{abstract}
In this work, the dissipative nature of spatially distributed process systems is exploited to develop efficient exponential state observers based on a low dimensional dynamic representation of the original set of partial differential equations. The approach we suggest combines standard observer design techniques for reactors where the reaction rates are unknown with efficient model reduction methodologies based on projection of the original concentration and temperature fields on low dimensional subspaces capturing the slow dynamics of the process. The global exponential stability of the resulting observer is derived combining classical Lyapunov analysis with a transformation that allows us to obtain a diffusion system from a diffusionconvection system. In addition aspects related to the location of sensors and their influence on the ability to reconstruct the necessary fields to feed the observer will also be considered.
\end{abstract}

Key words: Observer, Distributed Process Systems, Tubular (Bio)Reactors, Optimal sensor location.

\section{Introduction}

As pointed out by Bastin and Dochain ${ }^{1}$, on-line monitoring and control in biotechnology have been hampered by a number of obstacles essentially associated with the poor knowledge of the process and the lack of reliable sensors capable of providing on-line measurements of the biochemical variables. To

*

Email addresses: antonio@iim.csic.es, Phone: +34986231930 Ext. 251

(Antonio A. Alonso ).

URL: http://www.iim.csic.es/ gingproc/ (Antonio A. Alonso). 
overcome these limitations, a theoretical identification framework has been presented by Bastin and Dochain ${ }^{1}$, and extended by Dochain et al. ${ }^{2}$, which exploits the underlying reaction structure and transfer mechanisms of the biotechnological processes to systematically design and implement state or parameter identification schemes. This theory has been extended to particular classes of distributed reactors and bio-reactors ${ }^{3,4}$. However, a number of issues and questions related to the spatially distributed nature of this class of systems and convergence analysis still remain open.

In fact, on-line state observation is particularly critical in spatially distributed reactors due to the high dimensionality associated with the dynamic representation. In this class of systems, the mass and energy balances result into a nonlinear set of partial differential equations whose solution usually involves the integration of a large set of ordinary differential equations ${ }^{5}$. In addition the observer must be supplied with on-line state measurements covering the whole spatial domain which are usually unavailable due to the limited number of sensors ${ }^{4,6,7}$.

In order to overcome these issues, we develop a generalized version of the state observer proposed by Bastin and Dochain ${ }^{1}$ for distributed tubular reactors, proving the exponential convergence of the error depending on the design parameters via Lyapunov analysis. In addition, the results developed by Alonso and co-workers ${ }^{8,9}$ are adapted to exploit the dissipative nature of diffusionconvection reaction systems ${ }^{10,11}$ and derive an implementation of the observer based on a low dimensional dynamic representation of the original system of partial differential equations. This framework will also be useful in devising a systematic solution to the field reconstruction problem from a limited number of measurements for those states that feed the observer. To that purpose, we use the formalism proposed by García et al. ${ }^{12}$ where a generalized version of the optimal sensor problems is formulated by taking advantage of the finite element method $^{13}$.

The paper is structured as follows: first of all the generalization and error convergence analysis of distributed observers is presented. After this section the model reduction technique is described and applied to the design of lowdimensional observers and state reconstruction schemes from a reduced number of sensors. Moreover, aspects related to the observer behavior under such approximations are studied at the end of the section. Following, Continuous production of gluconic acid will be employed to illustrate and validate these techniques and, finally, the conclusions are summarized. 


\section{Observer Design}

As it was pointed out by Dochain ${ }^{14}$, on-line measurements such as dissolved oxygen concentration, temperature or flow rates are usually available whereas, concentrations of biomass and some products and reactants require the use of state observers due to the lack of cheap or reliable on-line sensors. In addition the reaction rates in bioprocesses are usually unknown and classical state estimators such as Luenberger or Kalman observers based on perfect knowledge of the model structure cannot be applied. An observer design that circumvent such limitations was developed by Dochain and co-workers ${ }^{2,1,14}$ for stirred tank reactors with asymptotic convergence depending on the dilution rate. The same idea was extended for the case of tubular reactors over the discretized version of the original Partial Differential Equations (PDEs) without going inside the convergence properties ${ }^{4}$. In the next section, we present and adapt this theory to spatially distributed tubular reactors, discuss the physical meaning and prove the exponential convergence.

\section{General dynamic representation of tubular reactors}

Tubular reactors are diffusion-convection-reaction systems described by a set of PDEs always transformable to the following general dynamic structure:

$$
\frac{\partial x}{\partial t}=D \frac{\partial^{2} x}{\partial \xi^{2}}-v \frac{\partial x}{\partial \xi}+K \varphi(x)+Q\left(x^{*}-x\right)
$$

where $x(t, \xi) \in \mathbb{R}^{s}$ represents the state vector field as a function of time $t \in$ $[0, \infty)$ and spatial coordinates $\xi \in[0, L] \equiv \Omega$ (where $L$ is the reactor length). $v \in \mathbb{R}^{+}$denotes flow velocity and $D, Q \in \mathbb{R}^{s \times s}$ are positive and semi-positive definite diagonal matrices which contain the parameters describing dispersion and exchange with the environment property included in vector $x^{*} \in \mathbb{R}^{s}$. The kinetic part is described by a vector of nonlinear functions describing reaction rates $\varphi(x) \in \mathbb{R}^{r}$ and a full column rank matrix $K \in \mathbb{R}^{s \times r}$ of yield coefficients ${ }^{1}$. Finally, the description is completed with appropriate boundary and initial conditions, which for tubular reactors with axial dispersion are:

$$
\begin{gathered}
D \frac{\partial x}{\partial \xi}=-v\left(x^{i n}-x\right) \quad \forall t \in \mathbb{R}^{+}, \xi=0, \\
\frac{\partial x}{\partial \xi}=0 \quad \forall t \in \mathbb{R}^{+}, \xi=L \\
\text { and } x=x^{0} \quad \forall \xi \in[0, L], t=0
\end{gathered}
$$

where $(2 \mathrm{a})$ and $(2 \mathrm{~b})$ are the so-called Danckwerts boundary conditions with $L$ being the longitudinal length of the reactor, $x^{i n}(t) \in \mathbb{R}^{s}$ the input and 
$x^{0}(\xi) \in \mathbb{R}^{s}$ the initial condition.

We should note that solutions for system (1) with natural boundary conditions (2) can be found in the Sobolev spaces:

$$
\begin{gathered}
H^{0}(\Omega) \equiv L^{2}(\Omega)=\left\{\mathrm{f}: \Omega \rightarrow \mathbb{R} \text { such as }\|\mathrm{f}\|_{\Omega}=\left(\int_{\Omega} \mathrm{f}^{2} d \xi\right)^{1 / 2}<\infty\right\} \\
H^{q}(\Omega)=\left\{\mathrm{f} \in H^{q-1}(\Omega): \frac{\mathrm{d} f}{\mathrm{~d} \xi} \in H^{q-1}(\Omega)\right\}
\end{gathered}
$$

being $q=1,2, \ldots$ and where $\mathrm{f}$ is a given function over the spatial domain $\Omega$ and over the boundary $\Gamma$.

State reconstruction without knowledge of the reaction rates

The development of distributed state observers which do not require knowledge of the reaction rates relies on the principles of mass and energy conservation which essentially state the existence of some unaltered entities collected on a vector $\bar{z}$ and related to the state vector $x$ by a linear map of the form $\bar{z}=B^{\mathrm{T}} x$. Since these entities are not produced, neither destroyed by reaction, but only transported through the system, the columns of $B$ must form a basis for the null space of $K^{\mathrm{T}}$ so that $B^{\mathrm{T}} K=0$. We should note that, since $K$ is column full rank $(r)$, the dimension of the $K$ left null space is $s-r$. This fact allows us to define the following partitions in $K$ and $B$, respectively:

$$
B^{\mathrm{T}}=\left[B_{e}^{\mathrm{T}} \mid B_{m}^{\mathrm{T}}\right] \quad K^{\mathrm{T}}=\left[K_{e}^{\mathrm{T}} \mid K_{m}^{\mathrm{T}}\right]
$$

where $K_{m}^{\mathrm{T}} \in \mathbb{R}^{r \times r}$ and $B_{e}^{\mathrm{T}} \in \mathbb{R}^{s-r \times s-r}$. In addition, since $B^{\mathrm{T}} K=0$ we also have that:

$$
B_{m}^{\mathrm{T}}=-B_{e}^{\mathrm{T}} K_{e} K_{m}^{-1}
$$

It should be stressed that the partition of $K^{\mathrm{T}}$ must be chosen in order to matrix $K_{m}$ be invertible. On the other hand, $\bar{z}$ can be written as:

$$
\bar{z}=B^{\mathrm{T}} x=B_{e}^{\mathrm{T}} x_{e}+B_{m}^{\mathrm{T}} x_{m}
$$

substituting (4) into (5) and re-ordering terms, we then get:

$$
\bar{z}=B_{e}^{\mathrm{T}}\left(x_{e}-K_{e} K_{m}^{-1} x_{m}\right)
$$

Finally, by defining $z=B_{e}^{-T} \bar{z}$ a new transformation is obtained of the form:

$$
z=x_{e}+A_{o} x_{m} \quad \text { with } \quad A_{o}=-K_{e} K_{m}^{-1}
$$

This transformation, that coincides with the one proposed by Bastin and Dochain $^{1,4}$, determines which states can be observed $x_{e} \in \mathbb{R}^{s-r}$ from measurements $x_{m} \in \mathbb{R}^{r}$, without knowledge of the reaction rates (as shown next, 
the evolution of $z$ is independent of them), provided that a reliable estimation $z$ is at hand. To that purpose, let us re-order and partition the original system as follows:

$$
\begin{gathered}
\frac{\partial x_{e}}{\partial t}=D_{e} \frac{\partial^{2} x_{e}}{\partial \xi^{2}}-v \frac{\partial x_{e}}{\partial \xi}+K_{e} \varphi(x)+Q_{e}\left(x_{e}^{*}-x_{e}\right) \\
\frac{\partial x_{m}}{\partial t}=D_{m} \frac{\partial^{2} x_{m}}{\partial \xi^{2}}-v \frac{\partial x_{m}}{\partial \xi}+K_{m} \varphi(x)+Q_{m}\left(x_{m}^{*}-x_{m}\right)
\end{gathered}
$$

with:

$$
D=\left[\begin{array}{cc}
D_{e} & 0 \\
0 & D_{m}
\end{array}\right] \quad K=\left[\begin{array}{c}
K_{e} \\
K_{m}
\end{array}\right] \quad Q=\left[\begin{array}{cc}
Q_{e} & 0 \\
0 & Q_{m}
\end{array}\right]
$$

where $D_{e}, Q_{e} \in \mathbb{R}^{s-r \times s-r}$ and $D_{m}, Q_{m} \in \mathbb{R}^{m \times m}$ are diagonal matrices whereas $K_{e} \in \mathbb{R}^{s-r \times r}$ and $K_{m} \in \mathbb{R}^{m \times r}$ are full submatrices of $K$.

Applying (7) to (8) and adding and subtracting the terms $D_{e} A_{o} \frac{\partial^{2} x_{m}}{\partial \xi^{2}}+Q_{e} A_{o}\left(x_{m}^{*}-\right.$ $\left.x_{m}\right)$ in the transformed PDE, and the terms $v\left(A_{o} x_{m}^{i n}+A_{o} x_{m}\right)$ in the transformed boundary condition at $\xi=0$, we obtain the following reaction rate independent set of PDEs for the new states $z$ :

$$
\begin{gathered}
\frac{\partial z}{\partial t}=D_{e} \frac{\partial^{2} z}{\partial \xi^{2}}-v \frac{\partial z}{\partial \xi}-Q_{e} z+h\left(x_{m}, x^{*}\right) \\
D_{e} \frac{\partial z}{\partial \xi}=v z+g\left(x_{m}, x^{i n}\right) \quad \forall t \in \mathbb{R}^{+}, \xi=0 \\
\frac{\partial z}{\partial \xi}=0 \quad \forall t \in \mathbb{R}^{+}, \xi=L \\
z=z^{0} \quad \forall \xi \in[0, L], t=0
\end{gathered}
$$

with

$$
\begin{gathered}
h\left(x_{m}, x^{*}\right)=\left(A_{o} D_{m}-D_{e} A_{o}\right) \frac{\partial^{2} x_{m}}{\partial \xi^{2}}+\left(A_{o} Q_{m}-Q_{e} A_{o}\right)\left(x_{m}^{*}-x_{m}\right)+Q_{e} z^{*}, \\
g\left(x_{m}, x^{i n}\right)=-v\left(D_{e} A_{o} D_{m}^{-1}-A_{o}\right)\left(x_{m}^{i n}-x_{m}\right)-v z_{k}^{i n} \\
z^{*}=x_{e}^{*}+A_{o} x_{m}^{*}, \quad z^{i n}=x_{e}^{i n}+A_{o} x_{m}^{i n} \quad \text { and } \quad z^{0}=x_{e}^{0}+A_{o} x_{m}^{0}
\end{gathered}
$$

Remark 1 When diffusivities in $D$ and transfer coefficients in $Q$ are the same for each state $(D=d I$ and $Q=q I$ where $I$ represents the identity matrix) since now $h\left(x_{m}, x^{*}\right)$ and $g\left(x_{m}, x^{\text {in }}\right)$ become zero, the dynamic structure of the reactor is drastically simplified.

In order to prove the exponential convergence of the error for each observed state, let us re-write the PDE model in the element-wise case with the subindex $k$ extended to the dimension of $z$ (number of states minus reaction rates):

$$
\frac{\partial z_{k}}{\partial t}=d_{k} \frac{\partial^{2} z_{k}}{\partial \xi^{2}}-v \frac{\partial z_{k}}{\partial \xi}-q_{k} z_{k}+h_{k}\left(x_{m}, x^{*}\right)
$$




$$
\begin{gathered}
d_{k} \frac{\partial z_{k}}{\partial \xi}=v z_{k}+g_{k}\left(x_{m}, x^{i n}\right) \quad \forall t \in \mathbb{R}^{+}, \xi=0, \\
\frac{\partial z_{k}}{\partial \xi}=0 \quad \forall t \in \mathbb{R}^{+}, \xi=L \\
z_{k}=z_{k}^{0}=x_{k}^{0}+A_{o k} x_{m}^{0} \quad \forall \xi \in[0, L], t=0
\end{gathered}
$$

in such a way that the functions $h_{k}\left(x_{m}, x^{*}\right)$ and $g_{k}\left(x_{m}, x^{i n}\right)$ are:

$$
\begin{gathered}
h_{k}\left(x_{m}, x^{*}\right)=\left(A_{o k} D_{m}-d_{k} A_{o k}\right) \frac{\partial^{2} x_{m}}{\partial \xi^{2}}+\left(A_{o k} Q_{m}-q_{k} A_{o k}\right)\left(x_{m}^{*}-x_{m}\right)+q_{k} z_{k}^{*} \\
g_{k}\left(x_{m}, x^{i n}\right)=-v\left(d_{k} A_{o k} D_{m}^{-1}-A_{o k}\right)\left(x_{m}^{i n}-x_{m}\right)-v z_{k}^{i n}
\end{gathered}
$$

with

$$
z_{k}^{*}=x_{k}^{*}+A_{o k} x_{m}^{*} \quad \text { and } \quad z_{k}^{i n}=x_{k}^{i n}+A_{o k} x_{m}^{i n}
$$

and where $k$ denotes the kth row for full matrices $\left(A_{o}\right)$ and column vectors $\left(z^{i n}, z^{*}, z, x_{e}^{i n}, x_{e}^{*}, x_{e}^{0}\right)$ and the kth diagonal element for diagonal matrices $(D, Q)$.

Assuming that transport terms are known we have that:

$$
\begin{gathered}
\frac{\partial \widehat{z}_{k}}{\partial t}=d_{k} \frac{\partial^{2} \widehat{z}_{k}}{\partial \xi^{2}}-v \frac{\partial \widehat{z}_{k}}{\partial \xi}-q_{k} \widehat{z}_{k}+h_{k}\left(x_{m}, x^{*}\right) \\
d_{k} \frac{\partial \widehat{z}_{k}}{\partial \xi}=v \widehat{z}_{k}+g_{k}\left(x_{m}, x^{i n}\right) \quad \forall t \in \mathbb{R}^{+}, \xi=0, \\
\frac{\partial \widehat{z}_{k}}{\partial \xi}=0 \quad \forall t \in \mathbb{R}^{+}, \xi=L \\
\widehat{z}_{k}=\widehat{z}_{k}^{0}=z_{k}-e_{k}^{0} \quad \forall \xi \in[0, L], t=0
\end{gathered}
$$

where $x_{m}$ represents the measurable states and $e_{k}^{0}=x_{k}^{0}-\widehat{x}_{k}^{0}$ are the errors due to the unknown transformed initial condition. Finally each of the observed states $x_{k}$ that belong to the vector $x_{e}$ is recovered by inverting the transformation (7):

$$
\widehat{x}_{k}=\widehat{z}_{k}-A_{o k} x_{m}
$$

The equation describing the error evolution between the real and the observed states as $e_{k}=x_{k}-\widehat{x}_{k}=z_{k}-\widehat{z}_{k}$ is constructed by combining (10) and (11) so that:

$$
\begin{gathered}
\frac{\partial e_{k}}{\partial t}=d_{k} \frac{\partial^{2} e_{k}}{\partial \xi^{2}}-v \frac{\partial e_{k}}{\partial \xi}-q_{k} e_{k} \\
d_{k} \frac{\partial e_{k}}{\partial \xi}=v e_{k} \quad \forall t \in \mathbb{R}^{+}, \xi=0 \\
\frac{\partial e_{k}}{\partial \xi}=0 \quad \forall t \in \mathbb{R}^{+}, \xi=L \\
e_{k}=e_{k}^{0} \quad \forall \xi \in[0, L], t=0
\end{gathered}
$$


The convergence properties of this observation scheme are summarized in the following proposition where integrals over the domain $\Omega$ and over the boundary $\Gamma$ are denoted respectively as $\langle\cdot, \cdot\rangle_{\Omega}$ and $\langle\cdot, \cdot\rangle_{\Gamma}$. In order to show convergence, the following result by book of Polyanin (page 59) ${ }^{16}$ will be employed.

Lemma 1 The following PDE systems:

$\frac{\partial \Upsilon}{\partial t}=d \Delta \Upsilon-\boldsymbol{v} \cdot \nabla \Upsilon-q \Upsilon+f(\boldsymbol{\xi}, t) \quad$ and $\quad \frac{\partial \bar{\Upsilon}}{\partial t}=d \Delta \bar{\Upsilon}+\exp (\delta t-\boldsymbol{\mu} \cdot \boldsymbol{\xi}) f(\boldsymbol{\xi}, t)$

are related by the transformation:

$$
\Upsilon=\exp (\boldsymbol{\mu} \cdot \boldsymbol{\xi}-\delta t) \bar{\Upsilon} \quad \boldsymbol{\mu}=\frac{\boldsymbol{v}}{2 d}>\boldsymbol{O}, \quad \delta=\frac{v^{2}}{4 d}+q>0
$$

where the bold letters denote vectors and $\boldsymbol{\xi}=\left[\xi_{1}, \xi_{2}, \xi_{3}\right]$.

Proof: The demonstration is straightforward by introducing the transformation into the diffusion-convection system and applying the chain rule.

Proposition 1 Let the evolution of $z_{k}$ and $\widehat{z}_{k}$ be described by (10) and (11), respectively. Then $\widehat{z}_{k}$ will converge exponentially to $z_{k}$ in the $L^{2}$ norm.

Proof: Let us denote for the sake of clarity, the dynamics of each element $e_{k}$ of the error vector as:

$$
\begin{gathered}
\frac{\partial e(\xi, t)}{\partial t}=d \frac{\partial^{2} e(\xi, t)}{\partial \xi^{2}}-v \frac{\partial e(\xi, t)}{\partial \xi}-q e(\xi, t) \\
d \frac{\partial e(0, t)}{\partial \xi}=v e(0, t), \quad \frac{\partial e(L, t)}{\partial \xi}=0, \quad e(\xi, 0)=e^{0}
\end{gathered}
$$

The state-space description of the PDE system (13) can be re-written as an infinite-dimensional system as follows:

$$
\frac{\mathrm{d} \tilde{e}}{\mathrm{~d} t}=A \tilde{e} \quad \tilde{e}=e^{0} \text { for } t=0
$$

where $A$ is a operator of the form:

$$
A f:=d \frac{\mathrm{d}^{2} \mathrm{f}}{\mathrm{d} \xi^{2}}-v \frac{\mathrm{df}}{\partial \xi}-q \mathrm{f}
$$

with domain:

$$
\mathbf{D}(A)=\left\{\mathrm{f} \in H^{2}(\Omega) ; d \frac{\mathrm{df}(0)}{\mathrm{d} \xi}-v \mathbf{f}(0)=\frac{\mathrm{d} \mathbf{f}(L)}{\mathrm{d} \xi}=0\right\}
$$


By Lemma 1, the error evolution can be expressed as the classical diffusion PDE:

$$
\frac{\partial \bar{e}(\xi, t)}{\partial t}=d \frac{\partial^{2} \bar{e}(\xi, t)}{\partial \xi^{2}}
$$

where

$$
e=\exp (\mu \xi-\delta t) \bar{e} \quad \mu=\frac{v}{2 d}>0, \quad \delta=\frac{v^{2}}{4 d}+q>0
$$

with boundary and initial conditions:

$$
\frac{\partial \bar{e}(0, t)}{\partial \xi}=\mu \bar{e}(0, t), \quad \frac{\partial \bar{e}(L, t)}{\partial \xi}=-\mu \bar{e}(L, t), \quad \bar{e}(\xi, 0)=\bar{e}^{0} .
$$

Now, let us define a Lyapunov function $\mathcal{V}=\frac{1}{2}\langle\bar{e}, \bar{e}\rangle_{\Omega}$ and compute its time derivative along (15a) so that:

$$
\frac{\partial \mathcal{V}}{\partial t}=\left\langle\bar{e}, \frac{\partial \bar{e}}{\partial t}\right\rangle_{\Omega}=d\left\langle\bar{e}, \frac{\partial^{2} \bar{e}}{\partial \xi^{2}}\right\rangle_{\Omega}
$$

From the divergence theorem and introducing the boundary conditions (15c), we obtain:

$$
\begin{gathered}
d\left\langle\bar{e}, \frac{\partial^{2} \bar{e}}{\partial \xi^{2}}\right\rangle_{\Omega}=d\left[\bar{e} \frac{\partial \bar{e}}{\partial \xi}\right]_{0}^{L}-d\left\|\frac{\partial \bar{e}}{\partial \xi}\right\|_{\Omega}^{2}= \\
=-d \mu\left[\bar{e}(L, t)^{2}+\bar{e}(0, t)^{2}\right]-d\left\|\frac{\partial \bar{e}}{\partial \xi}\right\|_{\Omega}^{2} \leq-d\left\|\frac{\partial \bar{e}}{\partial \xi}\right\|_{\Omega}^{2}
\end{gathered}
$$

Furthermore, with boundary conditions (15c) the following Poincaré inequality holds:

$$
\left\|\frac{\partial \bar{e}}{\partial \xi}\right\|_{\Omega}^{2} \geq \nu\|\bar{e}\|_{\Omega}^{2},
$$

where $\nu$ is a positive parameter ${ }^{17,18}$. Combining this inequality with (16) we then obtain:

$$
\dot{\mathcal{V}} \leq-2 \nu d \mathcal{V}
$$

whose explicit solution using the Gronwall-Bellman lemma is:

$$
\mathcal{V} \leq \mathcal{V}^{0} \exp (-2 d \nu t)
$$

that by using $\mathcal{V}=\frac{1}{2}\|\bar{e}\|_{\Omega}^{2}$ remains:

$$
\|\bar{e}\|_{\Omega}^{2} \leq\left\|\bar{e}^{0}\right\|_{\Omega}^{2} \exp (-2 d \nu t) .
$$

Finally, the $L^{2}$ norm of the observation error using relation (15b) is bounded as follows:

$$
\|e\|_{\Omega}^{2} \leq\|\exp (\mu \xi-\delta t)\|_{\Omega}^{2}\|\bar{e}\|_{\Omega}^{2} \equiv \varpi \exp [-2(\delta+d \nu) t]
$$

with

$$
\varpi=\frac{\exp (2 \mu L)-1}{2 \mu}\left\|\bar{e}^{0}\right\|_{\Omega}^{2}
$$


Remark 2 As it can be concluded from (17) the observation rate of convergence will depend on process design parameters such as the degree of dispersion, velocity and/or mass and energy transfer coefficients. In fact the larger the transfer coefficient $\left(q_{k}\right)$ and the reactor velocity $(v)$, and the lower the dispersion coefficient $\left(d_{k}\right)$, the faster the convergence.

We should note that if we have at our disposal a given number of sensors for measuring the field $x_{e}$ at some spatial locations, then classical estimators with manipulable velocity convergence, such as the Luenberger or Kalman observers $^{4,14,19}$, could be employed to reconstruct the whole field without knowledge of the reaction rates.

\section{Observer Implementation}

In order to implement the proposed observation scheme (11), the PDE set needs to be solved on-line and the complete field $x_{m}$ measured. Standard PDE solvers based on finite differences or finite element methods (FEM) essentially consist of spatial discretization schemes that approximate the original PDEs by an usually large set of ordinary differential equations (ODEs). The FEM, in particular, is specially advantageous because allows us to obtain the finite set of ODEs in a systematic way regardless of the geometry of the spatial domain. Although in this article we propose the use of reduced order models to obtain a low dimensional set of ODEs, the projections will be implemented by exploiting the underlying FEM algebraic structure which we summarize next:

The FEM method is based on the Galerkin projection of the original PDEs over a set of locally defined basis functions $\left\{\psi_{i}\right\}_{i=1}^{n}{ }^{13}$, known as finite element functions, plus a relaxation of the second derivatives using the divergence theorem. The resulting PDEs (11) are formally stated as the solution of the ODE set:

$$
\dot{Z}_{k}=\mathcal{D} \mathcal{A}^{-1}\left[-\left(d_{k} \mathcal{C}-v \mathcal{Q}+v \mathcal{B E}\right) Z_{k}+\mathcal{G}^{k}\right]+q_{k}\left(Z_{k}^{*}-Z_{k}\right)+H_{k}\left(x_{m}, x^{*}\right)
$$

where $Z_{k} \in \mathbb{R}^{n}$ and $H_{k}\left(x_{m}, x^{*}\right) \in \mathbb{R}^{n}$ correspond with the discrete versions of the original distributed functions $z_{k}$ and $h_{k}\left(x_{m}, x^{*}\right)$. On the other hand the FEM matrices are computed as ${ }^{13}$ :

$$
\mathcal{B E}_{i j}=\left\langle\frac{\partial \psi_{i}}{\partial \xi}, \psi_{j}\right\rangle_{\Omega} \quad \mathcal{C}_{i j}=\left\langle\frac{\partial \psi_{i}}{\partial \xi}, \frac{\partial \psi_{j}}{\partial \xi}\right\rangle_{\Omega}
$$




$$
\mathcal{D} \mathcal{A}_{i j}=\left\langle\psi_{i}, \psi_{j}\right\rangle_{\Omega} \quad \mathcal{Q}_{i j}=\left\langle\psi_{i}, \psi_{j}\right\rangle_{\Gamma} \quad \mathcal{G}_{j}^{k}=\left\langle g_{k}\left(x_{m}, x^{i n}\right), \psi_{j}\right\rangle_{\Gamma}
$$

Alternatively to the step-by-step Galerkin projection, the discretized version (18) of the PDE system (11a) can be obtained in a straightforward way by means of the FEM equivalents to infinite dimensional operators. Table 1 shows the mappings between the continuous and discrete domain integrals, gradients or Laplacians for a pair of arbitrary functions $f$ and $g$ continuous in space, where $\mathrm{F}$ and $\mathrm{G}$ correspond to the discretized version of such functions. More details about the exploitation of the FEM structure to obtain the relations for $1 \mathrm{D}, 2 \mathrm{D}$ or $3 \mathrm{D}$ spatial operators can be seen in the work of García et al. ${ }^{12}$.

As pointed out by Christofides ${ }^{5}$ and Delattre and co-workers ${ }^{20}$, approaches based on spatial discretization present a number of disadvantages: they are usually computationally involved and some essential control-theoretic properties, such as controllability or observability, may be lost by the discretization scheme or the degree of refinement. Moreover, complete measurements of the field $x_{m}$ are not usually available due to the large number of sensors required, which calls for efficient field reconstruction schemes from a reduced number of sensors ${ }^{6}$. These questions will be properly addressed next in the framework of reduced order modelling (ROM) of dissipative systems ${ }^{7,12}$.

In constructing a reduced order dynamic representation for any field $x(t, \xi)$ with general dynamic structure (1), we make use of the dissipative nature of diffusion-convection systems and expand the field $x(t, \xi)$ as a complete series of orthonormal globally defined basis functions $\left\{\phi_{i}^{x}(\xi)\right\}_{i=1}^{\infty}$ and time dependent functions $\left\{c_{i}^{x}(t)\right\}_{i=1}^{\infty}$ so that:

$$
x(t, \xi)=\sum_{i=1}^{\infty} c_{i}^{x}(t) \phi_{i}^{x}(\xi)
$$

where each $\phi_{i}^{x}$ is computed by solving the following eigenvalue problem:

$$
\int_{\Omega} R\left(\xi, \xi^{\prime}\right) \phi_{i}^{x}\left(\xi^{\prime}\right) \mathrm{d} \xi^{\prime}=\lambda_{i}^{x} \phi_{i}^{x}(\xi)
$$

with $\lambda_{i}^{x}$ being the eigenvalue associated to each eigenfunction $\phi_{i}^{x}$ of the field $x$. Depending on the nature of the kernel $R$ different sets of basis functions emerge $^{7}$, among which the following are considered:

(1) Laplacian Spectral Decomposition (LSD), where $R$ is the Green function associated with a symmetric spatial operator.

(2) Proper Orthogonal Decomposition (POD), where $R$ is a two point correlation matrix constructed from empirical data (snapshots).

In both cases, the ordered structure of the eigenspectrum $\left\{\lambda_{i}^{x}(\xi)\right\}_{i=1}^{\infty}$ defines a set of low dimensional subspaces $\left\{\phi_{i}^{x}(\xi)\right\}_{i=1}^{m_{x}}$ which approximate the original 
field, thus guiding the selection of the subspace which captures most of the relevant dynamic features of the solution. This fact was proved by Christofides and Daoutidis ${ }^{21}$ for the LSD and by Baker and Christofides ${ }^{22}$ for the POD method using the concept of approximate inertial manifolds. Once the subspace is defined, the field is approximated by a truncated series expansion of the form:

$$
x(t, \xi) \cong \tilde{x}(t, \xi)=\sum_{i=1}^{m_{x}} c_{i}^{x}(t) \phi_{i}^{x}(\xi)
$$

In the following sections, the LSD will be employed to expand the observer PDEs (11) while the POD method will be used for the reconstruction of the measurable states from partial measurements. Finally, in the last section, the final estimation error due to the latter approximations plus the influence of the sampling period will be considered.

\section{Reduced order observer representation}

Similar to the FEM method, the low dimensional dynamic representation is constructed by projecting the observer (11) using global basis functions obtained from the LSD, instead of the local finite element functions. However the Galerkin's method combined with global basis is only applicable under homogeneous boundary conditions and, therefore, only when no perturbations in the inlet are taken into account. To avoid such restriction two alternatives are in hand whose advantages and disadvantages can be seen in Balsa et al. ${ }^{23}$ : the tau-method ${ }^{24}$, and the transformation of the original problem into an homogeneous boundary system ${ }^{25,26}$. In this work we make use of the second alternative, namely that in which the non-homogeneous boundary conditions (11b) are transformed into their homogeneous equivalents by using the following linear transformation:

$$
p_{k}\left(x_{m}\right)=z_{k}\left(x_{m}\right)+\left.\frac{1}{v} g_{k}\left(x_{m}, x^{i n}\right)\right|_{\xi=0}
$$

where $\left.g_{k}\left(x_{m}, x^{i n}\right)\right|_{\xi=0}$ is a time-depending function that corresponds with the equation (10f) evaluated in the first point of the reactor $(\xi=0)$ :

$$
\begin{gathered}
\left.g_{k}\left(x_{m}, x^{i n}\right)\right|_{\xi=0}=g_{k}\left(x_{m}(0, t), x^{i n}(t)\right)= \\
=-v\left(d_{k} A_{o k} D_{m}^{-1}-A_{o k}\right)\left(x_{m}^{i n}(t)-x_{m}(0, t)\right)-v z_{k}^{i n}(t)
\end{gathered}
$$

The evolution of the transformed field $p_{k}$ is obtained by computing its time derivative along (10) so that:

$$
\frac{\partial p_{k}}{\partial t}=d_{k} \frac{\partial^{2} p_{k}}{\partial \xi^{2}}-v \frac{\partial p_{k}}{\partial \xi}-q_{k} p_{k}+f_{k}\left(x_{m}, x^{*}, x^{i n}\right)
$$




$$
\begin{gathered}
d_{k} \frac{\partial p_{k}}{\partial \xi}=v p_{k} \quad \forall t \in \mathbb{R}^{+}, \xi=0, \\
\frac{\partial p_{k}}{\partial \xi}=0 \quad \forall t \in \mathbb{R}^{+}, \xi=L \\
p_{k}=p_{k}^{0} \quad \forall \xi \in[0, L], t=0 \\
f_{k}\left(x_{m}, x^{*}, x^{i n}\right)=\left.\frac{q_{k}}{v} g_{k}\left(x_{m}, x^{i n}\right)\right|_{\xi=0}+h_{k}\left(x_{m}, x^{*}\right)+\left.\frac{1}{v} \dot{g}_{k}\left(x_{m}, x^{i n}\right)\right|_{\xi=0}
\end{gathered}
$$

In the same way, the observer of this transformed field $p_{k}$, computed from (11), takes the form:

$$
\begin{gathered}
\frac{\partial \widehat{p}_{k}}{\partial t}=d_{k} \frac{\partial^{2} \widehat{p}_{k}}{\partial \xi^{2}}-v \frac{\partial \widehat{p}_{k}}{\partial \xi}-q_{k} \widehat{p}_{k}+f_{k}\left(x_{m}, x^{*}, x^{i n}\right) \\
d_{k} \frac{\partial \widehat{p}_{k}}{\partial \xi}=v \widehat{p}_{k} \quad \forall t \in \mathbb{R}^{+}, \xi=0 \\
\frac{\partial \widehat{p}_{k}}{\partial \xi}=0 \quad \forall t \in \mathbb{R}^{+}, \xi=L \\
\widehat{p}_{k}=\widehat{p}_{k}^{0}=p_{k}^{0}-e_{k}^{0} \quad \forall \xi \in[0, L], t=0 \quad \text { with } \quad e_{k}^{0}=x_{k}^{0}-\widehat{x}_{k}^{0}
\end{gathered}
$$

where the estimated concentrations and temperatures are recovered by combining the transformation (11e) and (23) and re-arranging terms:

$$
\widehat{x}_{k}\left(x_{m}\right)=\widehat{p}_{k}\left(x_{m}\right)-\left.\frac{1}{v} g_{k}\left(x_{m}, x^{i n}\right)\right|_{\xi=0}-A_{o k} x_{m}
$$

Instead of using the classical FEM to solve the latter PDE system we will employ the ROM approach where, similarly with equation (22), the field $\widehat{p}_{k}$ is approximated by a truncated series expansion of the form:

$$
\widehat{p}_{k} \cong \tilde{p}_{k}=\sum_{i=1}^{m_{p_{k}}} \widehat{c}_{i}^{p_{k}} \phi_{i}^{p_{k}}
$$

with $m_{p_{k}}$ denoting the dimension of the subspace chosen. Global basis functions $\left\{\phi_{i}^{p_{k}}(\xi)\right\}_{i=1}^{m_{p_{k}}}$ are obtained from the eigenvalue problem (21) associated with the diffusion operator in equation (26) (see the Courant and Hilbert book $^{27}$ for details):

$$
\begin{gathered}
\frac{\mathrm{d}^{2} \phi_{i}^{p_{k}}(\xi)}{\mathrm{d} \xi^{2}}=-\lambda_{i}^{p_{k}} \phi_{i}^{p_{k}}(\xi) \\
d_{k} \frac{\mathrm{d} \phi_{i}^{p_{k}}}{\mathrm{~d} \xi}=v \phi_{i}^{p_{k}} \quad \forall t \in \mathbb{R}^{+}, \xi=0 \\
\frac{\mathrm{d} \phi_{i}^{p_{k}}}{\mathrm{~d} \xi}=0 \quad \forall t \in \mathbb{R}^{+}, \xi=L
\end{gathered}
$$


and time dependent functions $\left\{\widehat{c}_{i}^{p_{k}}(t)\right\}_{i=1}^{m_{p_{k}}}$ by projecting the transformed observed state (26a) over $\left\{\phi_{i}^{p_{k}}(\xi)\right\}_{i=1}^{m_{p_{k}}}$. Noting that such functions are orthonormal, the following ODE set is obtained:

$$
\frac{\mathrm{d} \widehat{c}_{i}^{p_{k}}}{\mathrm{~d} t}=-d_{k} \lambda_{i}^{p_{k}} \widehat{c}_{i}^{p_{k}}+\int_{\Omega} \phi_{i}^{p_{k}}\left(-v \frac{\partial \widehat{p}_{k}}{\partial \xi}-q_{k} \widehat{p}_{k}+f_{k}\left(x_{m}, x^{*}, x^{i n}\right)\right) \mathrm{d} \xi
$$

For computational purposes, let us express both eigenvalue (29a) and initialboundary (30) problems in their discrete versions using the FEM matrices according to Table 1 . Therefore, the off-line computation of the spatial basis $\left\{\phi_{i}^{p_{k}}(\xi)\right\}_{i=1}^{m_{p_{k}}}$ is obtained from:

$$
\left(d_{k} \mathcal{C}+v \mathcal{Q}\right) \Phi_{i}^{p_{k}}=-\lambda_{i}^{p_{k}} \mathcal{D} \mathcal{A} \Phi_{i}^{p_{k}} \quad \text { with } \quad i=1, . ., m_{p_{k}}
$$

where each $\Phi_{i}^{p_{k}} \in \mathbb{R}^{n}$ represents the spatial discrete version of $\phi_{i}^{p_{k}}$. On the other hand, the initial-boundary problem (30) is solved on-line in its reduced order version:

$$
\begin{gathered}
\frac{\mathrm{d} \widehat{C}^{p_{k}}}{\mathrm{~d} t}=\left(-d_{k} \Lambda^{p_{k}}+\Phi^{p_{k} \mathrm{~T}}\left(-v \mathcal{B} \mathcal{E}-q_{k} \mathcal{D} \mathcal{A}\right) \Phi^{p_{k}}\right) \widehat{C}^{p_{k}}+\Phi^{p_{k} \mathrm{~T}} \mathcal{D} \mathcal{A} f_{k}\left(x_{m}, x^{*}, x^{i n}\right) \\
\widehat{C}^{p_{k}}(t)=\Phi^{p_{k} \mathrm{~T}} \mathcal{D} \mathcal{A} \widehat{p}_{k}(t) \quad \forall \xi \in[0, L], t=0
\end{gathered}
$$

where $\Lambda^{p_{k}} \in \mathbb{R}^{m_{p_{k}} \times m_{p_{k}}}$ is a diagonal matrix containing the eigenvalues of equation (31) and $\Phi^{p_{k}}=\left[\Phi_{1}^{p_{k}}, \ldots, \Phi_{m_{p_{k}}}^{p_{k}}\right] \in \mathbb{R}^{n \times m_{p_{k}}}$ and $\widehat{C}^{p_{k}}(t)=\left[\widehat{C}_{1}^{p_{k}}(t), \ldots, \widehat{C}_{m_{p_{k}}}^{p_{k}}(t)\right] \in$ $\mathbb{R}^{m_{p_{k}}}$ collect in matrix and vector form, respectively, the sets $\left\{\phi_{i}^{p_{k}}(\xi)\right\}_{i=1}^{m_{p_{k}}}$ and $\left\{\hat{c}_{i}^{p_{k}}(t)\right\}_{i=1}^{m_{p_{k}}}$. Finally, the estimation of concentrations and temperatures $\widehat{x}_{e}$ at every point of the FEM mesh $\left(\widehat{X}_{e}\right)$ can be recovered from:

$$
\widehat{X}_{e}=\tilde{P}(t)-\left.\frac{1}{v} g\left(X_{m}, x^{i n}\right)\right|_{\xi=0}-A_{o} X_{m}
$$

with:

$$
\tilde{P}(t)=\left[\Phi^{p_{1}} \widehat{C}^{p_{1}}(t), \ldots, \Phi^{p_{s-r}} \widehat{C}^{p_{s-r}}(t)\right]^{\mathrm{T}} \in \mathbb{R}^{s-r \times n} \quad \text { and } \quad X_{m} \in \mathbb{R}^{r \times n}
$$

Optimal field reconstruction from a limited set of measurements

As we have mentioned before, observer (11) implemented either in the FEM framework (18) or as a reduced order observer (32) requires on-line measurements of the complete field $x_{m}$ which are not usually available. In addition, the PDE set that describes the behavior of the measurable states (8b) depends on the unknown reaction rates so a dynamic ROM is not possible.

Although the need for systematic selection of optimal sensor location, as well as inputs and outputs pairings in distributed systems has become evident ${ }^{28}$, 
exhaustive search procedures which can be useful for placing a small number of sensors are still widely employed. Alternative approaches include the one proposed by Antoniades and Christofides ${ }^{29}$ to solve the placement problem by standard unconstrained optimization and taking advantage of the time scale separation properties of transport-reaction systems. The approach, although elegant, requires the process to be under control and restricts the number of sensors to be equal to the dimension of the slow dynamics. A theory that circumvents such limitations was proposed by Alonso and co-workers ${ }^{7}$ for pointwise sensors and extended by García et al. ${ }^{12}$ for both point-wise or array sensors. In these articles the empirical global basis obtained from the POD method are used to set up the location of sensors for continuous distributed processes and optimally reconstruct the complete field from a limited number of measurements. Details about the methodology applied to the estimation of the on-line measurable vector field $x_{m}$ are summarized in Appendix A.

Observer stability under the influence of the implementation approximations

In order to understand the behavior of the observer under the approximations proposed in previous subsections (reconstruction of the measurable field and reduced order model) as well as the influence of the sampling time, let us define the following error sources:

- Truncation error The truncation error associated with each of the $\tilde{p}_{k}$ fields is of the form:

$$
\epsilon_{k}=\widehat{p}_{k}-\tilde{p}_{k}=\sum_{i=m_{p_{k}}+1}^{\infty} \widehat{c}_{i}^{p_{k}} \phi_{i}^{p_{k}}, \quad \text { with } \quad k=1, \ldots, s-r
$$

- Reconstruction error The vector $x_{m}$ is only measured at some sampling times $\left\{t_{j}\right\}_{j=1}^{N_{s}}$ with a reduced number of optimal located sensors. At each $t_{j}$ the reconstruction of the field in the whole spatial domain, we denote by $\widehat{x}_{m}\left(t_{j}, \xi\right)$, is computed by using the methodology developed in appendix A. Linear behavior is considered between successive sampling times and therefore between successive reconstructions, so that:

$$
\widehat{x}_{m}(t, \xi)=a t+b \quad t \in\left[t_{j}, t_{j+1}\right]
$$

where

$$
a=\frac{\widehat{x}_{m}\left(t_{j+1}, \xi\right)-\widehat{x}_{m}\left(t_{j}, \xi\right)}{t_{j+1}-t_{j}}, \quad b=\widehat{x}_{m}\left(t_{j}, \xi\right)-a t_{j}
$$

The error associated with these approximations, we call reconstruction error in contrast to the observation error associated with the observed field $x_{e}$, is then represented as:

$$
e_{m}=x_{m}(t, \xi)-\widehat{x}_{m}(t, \xi), \quad \forall t, \xi
$$


In order to show the final convergence of the observer, we note that both the reconstruction error $e_{m}(t, \xi)$ and its second spatial derivative $\frac{\partial^{2} e_{m}}{\partial \xi^{2}}$ are bounded provided that the number of sensors is equal or greater than the number of the low-dimensional subspace used to represent $x_{m}$ (see Appendix A for details).

The following proposition establishes the region around the "true" field where the observations will exponentially converge. This region will depend on the truncation and reconstruction error.

Proposition 2 Let each of the process fields included in $x_{e}$ be described by equation (11e)

$$
x_{k}\left(x_{m}\right)=z_{k}\left(x_{m}\right)-A_{o k} x_{m}
$$

and the observations be obtained from equations (27) and (28) fed with $\widehat{x}_{m}$ :

$$
\widehat{x}_{k}\left(\widehat{x}_{m}\right)=\tilde{p}_{k}\left(\widehat{x}_{m}\right)-\left.\frac{1}{v} g_{k}\left(\widehat{x}_{m}, x^{i n}\right)\right|_{\xi=0}-A_{o k} \widehat{x}_{m}
$$

Therefore the norm of the observation errors $\left\|e_{k}\right\|_{\Omega}=\left\|x_{k}\left(x_{m}\right)-\widehat{x}_{k}\left(\widehat{x}_{m}\right)\right\|_{\Omega}$ will exponentially converge to a region defined by:

$$
\Xi_{k}=\eta_{k}+\left\|\left.\frac{1}{v} g_{k}\left(e_{m}\right)\right|_{\xi=0}+\epsilon_{k}-A_{o k} e_{m}\right\|_{\Omega}
$$

with

$$
\eta_{k}=\frac{F_{k}}{d_{k} \nu_{k}+\delta_{k}}\left[\frac{\exp \left(2 \mu_{k} L\right)+\exp \left(-2 \mu_{k} L\right)-2}{4 \mu_{k}^{2}}\right]^{1 / 2} \quad \text { and } \quad F_{k} \geq f_{k}\left(e_{m}\right)
$$

Proof: See Appendix B

Remark 3 When no implementation errors are considered, Proposition 2 coincides with Proposition 1 and the observations goes exponentially to the "true" states.

Remark 4 As it can be concluded from (39), the region where the observer will converge exponentially is a function of reconstruction error of the measurable variables $\left(e_{m}\right)$ and the truncation error. In fact we should note that, small relative errors in $\widehat{x}_{m}$ are translated into high percentage errors in the observed field $\widehat{x}_{e}$ when the order of magnitude of the measurements is significantly larger than the observations. 


\section{Case study: Gluconic Acid Production}

In order to illustrate the methodology developed in previous sections, we consider a tubular reactor for Gluconic Acid production whose biological features has been largely studied by Mirón and et al. ${ }^{30}$.

Gluconic Acid Dynamic Model for control and identification

The process takes place in a tubular reactor fed with glucose and oxygen. The consumption of glucose $(\mathrm{G})$ by the microorganisms $(X)$ to produce gluconic acid $(G A)$ motivates the following simplified mechanism:

$$
\begin{gathered}
G+X \stackrel{R_{X}}{\longrightarrow} X \\
G+\frac{1}{2} O_{2} \stackrel{R_{G A}}{\longrightarrow} G A
\end{gathered}
$$

where the biomass and gluconic acid reaction rates correspond with:

$$
\begin{gathered}
R_{X}=\mu^{X} X \frac{k_{1}^{X}-X}{k_{1}^{X}} ; \quad \mu^{X}=\frac{\mu_{\max }^{X} G}{k_{2}^{X}+G} \\
R_{G A}=\mu^{G A} G A \frac{k_{1}^{G A}-G A}{k_{1}^{G A}} ; \quad \mu^{G A}=\frac{\mu_{\max }^{G A} G}{k_{2}^{G A}+G}
\end{gathered}
$$

In accordance with the general dynamic structure proposed in (1) the state vector field, yield matrix and reaction kinetic vector is formally written as:

$$
x=\left[\begin{array}{c}
X \\
G A \\
G \\
O_{2}
\end{array}\right] K=\left[\begin{array}{cc}
1 & 0 \\
0 & 1 \\
-1 & -1 \\
0 & -0.5
\end{array}\right] \quad \varphi(x)=\left[\begin{array}{c}
R_{X} \\
R_{G A}
\end{array}\right]
$$

with oxygen mass exchange with the environment so that $Q$ is a null matrix except for the last element $Q(4,4)=k l a$.

Given the design parameters and feed streams of Table 2, the evolution and distribution of the concentrations under the perturbations in the glucose inlet depicted in Figure 1, are shown in Figure 2. 
The observation scheme for continuous gluconic acid production is designed, as discussed in previous sections, to produce estimates of biomass and gluconic acid from a limited number of measurements of oxygen and glucose so that according to (8) and (43) we have:

$$
\begin{gathered}
x_{e}=\left[\begin{array}{c}
X \\
G A
\end{array}\right] \quad x_{m}=\left[\begin{array}{c}
G \\
O_{2}
\end{array}\right] \\
K_{e}=\left[\begin{array}{ll}
1 & 0 \\
0 & 1
\end{array}\right] \quad K_{m}=\left[\begin{array}{cc}
-1 & -1 \\
0 & -0.5
\end{array}\right]
\end{gathered}
$$

The rest of matrices are null except the one that includes the oxygen exchange parameter:

$$
Q_{m}=\left[\begin{array}{cc}
0 & 0 \\
0 & k l a
\end{array}\right]
$$

In order to illustrate the theoretical performance of the observer developed in Proposition 1 without any approximation, let us consider a 15\% of error in the initial concentration of the products, perturb the glucose inlet as illustrated in Figure 1 and measure the input variables (Glucose and Oxygen) at any time and point of the spatial domain. The dynamic observer (11) is implemented using the FEM (18) with a mesh of 61 nodes. In Figure 3, the gluconic acid and biomass concentration errors are shown at any time along the reactor, illustrating the exponential-type error convergence property discussed in Proposition 1.

At this point, let us illustrate how the observed variables (biomass and gluconic acid) are affected by the model reduction and errors due to the sampling time and reconstruction from a limited number of sensors in the spatial domain. As it was commented in remark 2, special attention must be paid in the case of the biomass observation, obtained from:

$$
X=Z_{1}-G+2 O_{2}
$$

where small relative errors in $G$ and $Z_{1}$ fields would produce big relative errors in the biomass estimation, due to the differences of one order of magnitude between the glucose and the biomass. The gluconic acid estimation, however, does not present such inconvenient:

$$
G A=Z_{2}-2 O_{2}
$$


Anyway, and as we will show later, even in this case the methodology proposed can be applied being careful with the approximations considered.

In fact, when the reduced order framework is implemented, the error in the gluconic acid estimation has almost the same performance as the FEM implementation whereas the error in the biomass is slightly affected as shown Figure 4. For that purpose 7 and 8 basis functions computed from (31) were used for, respectively, gluconic acid and biomass. The ROM proposed, equations (28) and (33), represents a considerable reduction in the computational effort (20 ODEs in front of the 122 ODEs necessary when FEM is implemented), specially advantageous not also for observation, but also for control and optimization.

So far we have considered time-continuously oxygen and glucose measurements along the axial dimension of the reactor. In Figure 5 biomass relative error is depicted when the observer is fed with measurements every half an hour assuming linear behavior, equation (35a), between successive sampling times.

The appropriate number of sensors and optimal locations for glucose and oxygen measurements in the reactor, were selected as discussed in Appendix A from a POD basis set computed by solving (A.2) from a sufficiently rich set of dynamic snapshots. The POD set consisted of four and three elements for glucose and oxygen concentrations, respectively. The optimal location of sensors was obtained by solving the max-min problem (A.7) with the guided search algorithm developed by Alonso and co-workers ${ }^{7}$. The locations along the reactor of the five and four sensors for glucose (triangles) and oxygen (circles) are depicted in Figure 6.

Their measurements were employed to reconstruct the whole field by means of equations (A.1) and (A.6). As shown in Figure 7 for glucose and oxygen concentration, the reconstruction error remains extremely low in the event of perturbations thus showing good reconstruction properties.

Finally, the error obtained combining all the approximations of the proposed methodology can be seen in Figure 8 where the differences between the observation and the state, 250 hours after the initialization, remain in both cases below $3.5 \%$ (see figure 9) of relative error despite the ill-conditioned estimation of biomass.

\section{Conclusions}

In this work, a novel systematic observer design methodology for spatially distributed continuous reactors was presented. The approach, which extends 
further the theory of reactor estimation developed by Bastin and Dochain to diffusion-convection-reaction processes, takes advantage of its dissipative nature to demonstrate the exponential convergence depending on the design parameters and derive robust and efficient low order dimensional observation schemes. In addition, practical aspects of importance in industrial implementation such as the optimal location of a given limited number of sensors can be also easily handled.

\section{Acknowledgements}

Acknowledgments This paper includes results of the joint research project that is supported by the bilateral agreements between Bulgarian Academy of Sciences and Process Engineering Group IIM-CSIC. The authors acknowledge financial support received from the Spanish Government (MCyT Projects PPQ2001-3643 and DPI2004-07444-C04-03) and Xunta de Galicia (PGIDIT02PXIC40209PN). 


\section{List of Figures}

1 Perturbations in the Glucose inlet

2 Evolution and distribution of the relevant states

3 Error convergence (\%) using the FEM with measurements at any time and point of the spatial domain

4 error convergence (in percentage) using ROM with measurements at any time and spatial point

5 Error (in percentage) convergence using ROM with measurements every 30 minutes along the whole reactor

6 Optimal placement of sensors for glucose (triangles) and oxygen (circles) in the reactor

$7 \quad$ Error (in percentage) in the reconstruction using a limited number of sensors for glucose and oxygen

8 Evolution of the ROM observation error in the estimation of gluconic acid and biomass from partial measurements

9 Distribution of the ROM observation error in the estimation of gluconic acid and biomass from partial measurements at final time 


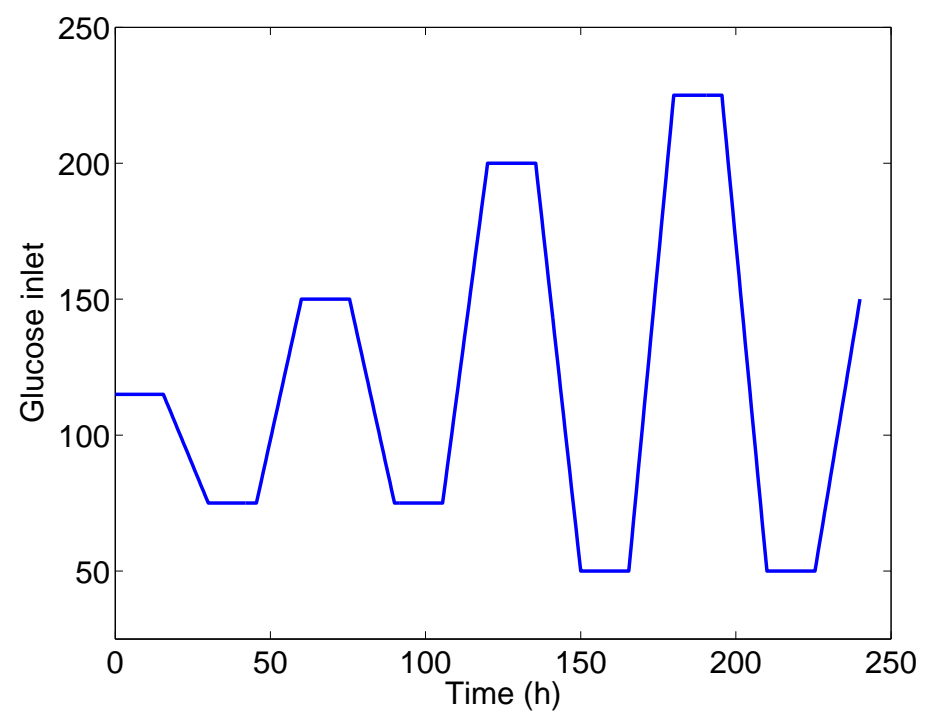

Fig. 1. Perturbations in the Glucose inlet 

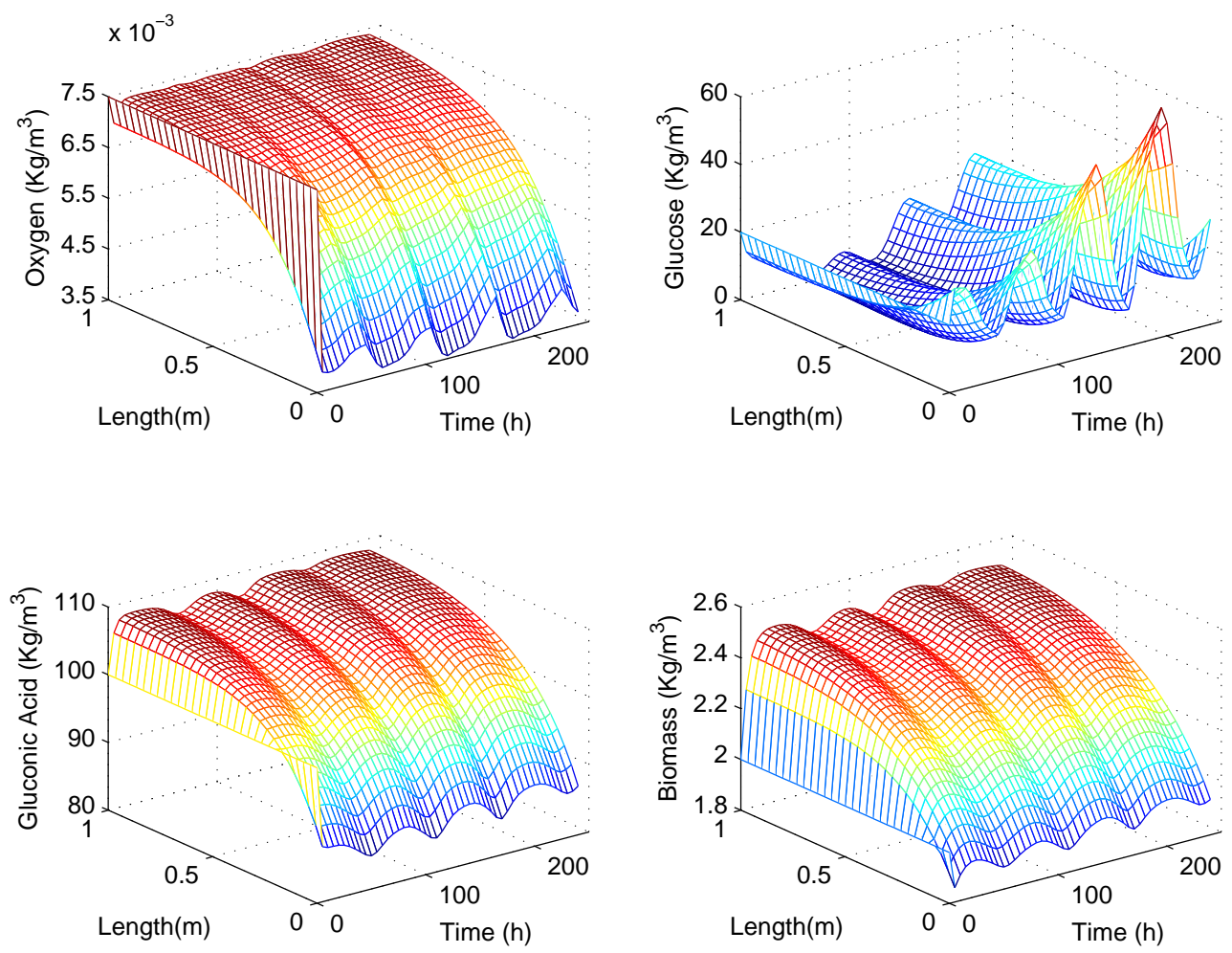

Fig. 2. Evolution and distribution of the relevant states 

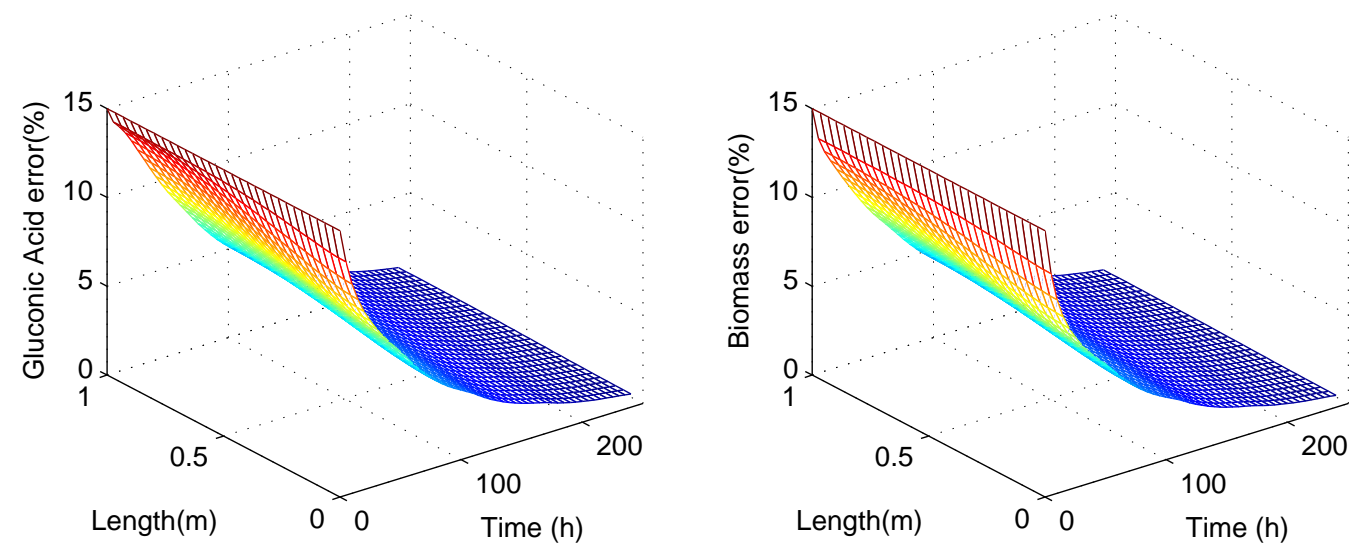

Fig. 3. Error convergence (\%) using the FEM with measurements at any time and point of the spatial domain 


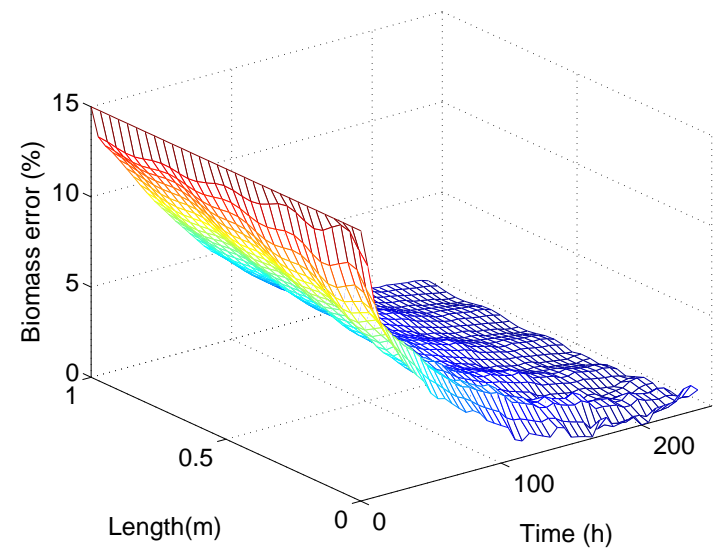

Fig. 4. error convergence (in percentage) using ROM with measurements at any time and spatial point 

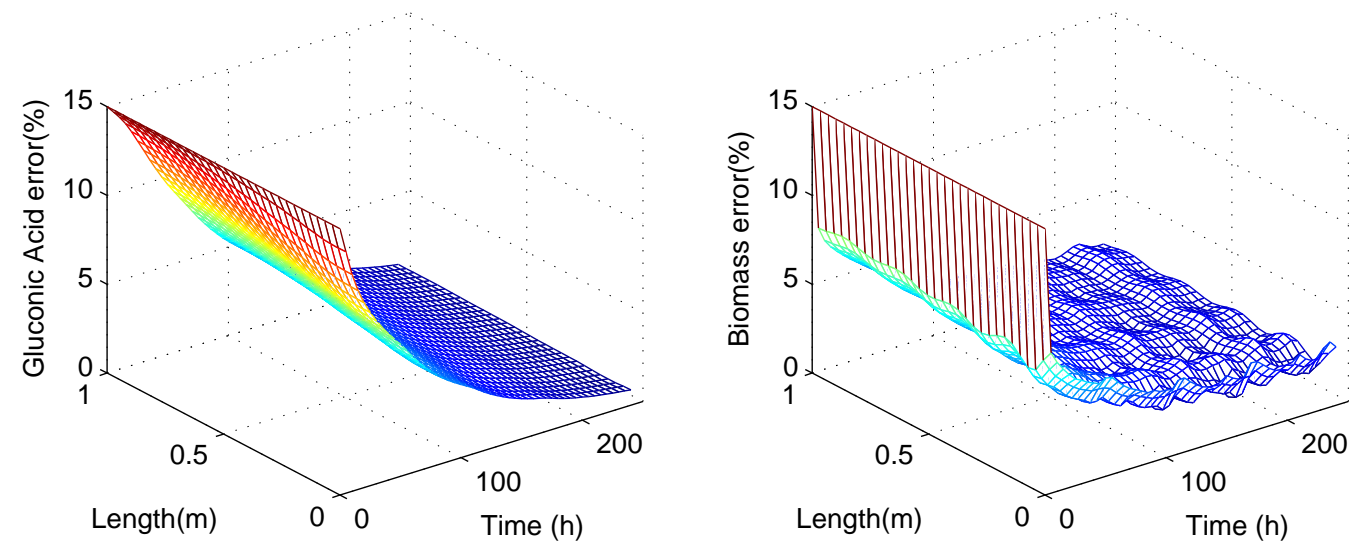

Fig. 5. Error (in percentage) convergence using ROM with measurements every 30 minutes along the whole reactor 


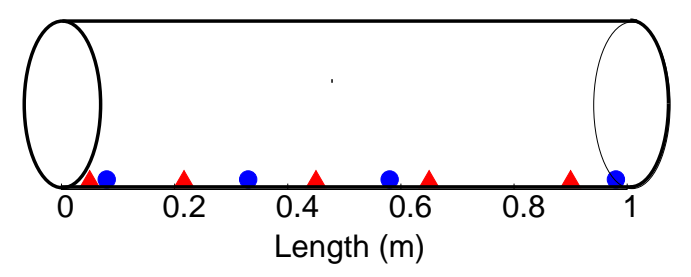

Fig. 6. Optimal placement of sensors for glucose (triangles) and oxygen (circles) in the reactor 

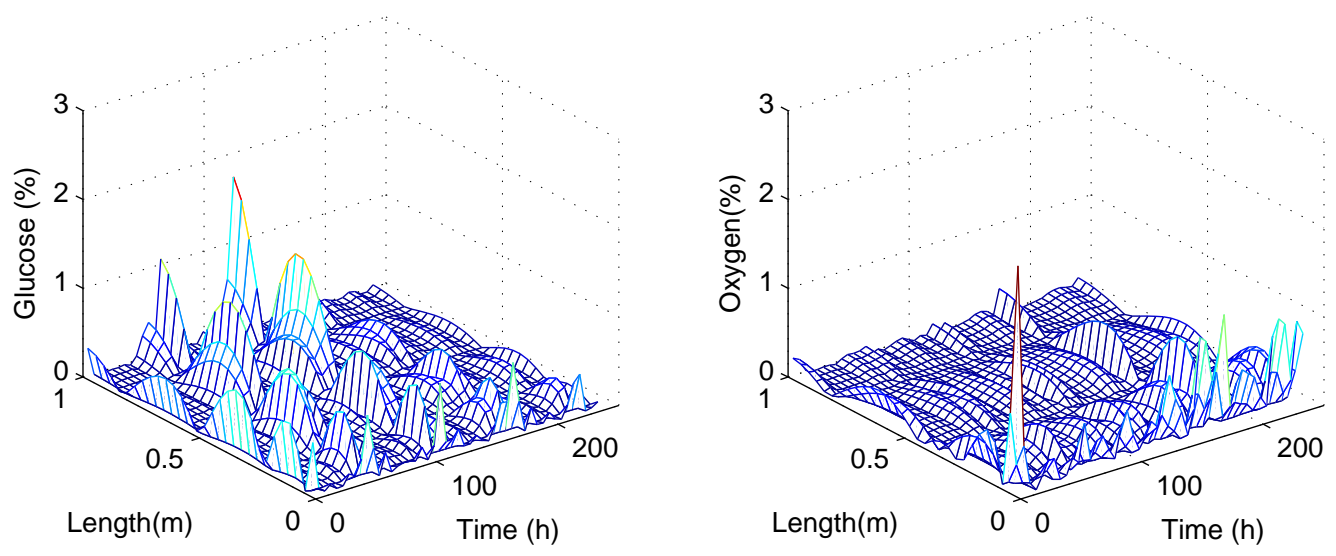

Fig. 7. Error (in percentage) in the reconstruction using a limited number of sensors for glucose and oxygen 

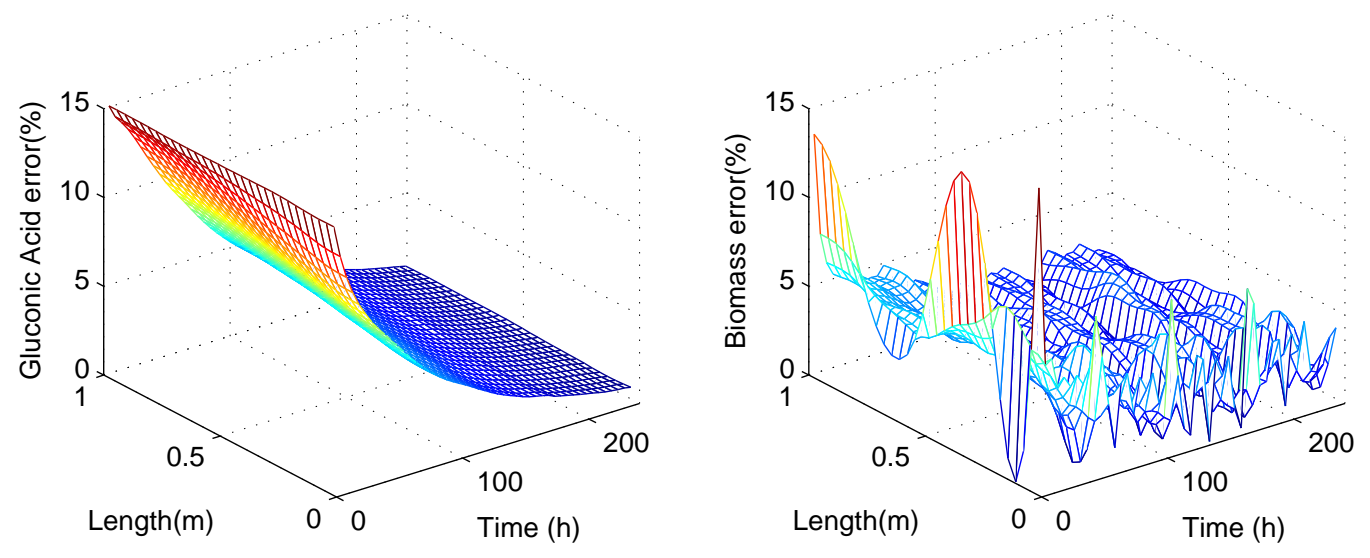

Fig. 8. Evolution of the ROM observation error in the estimation of gluconic acid and biomass from partial measurements 

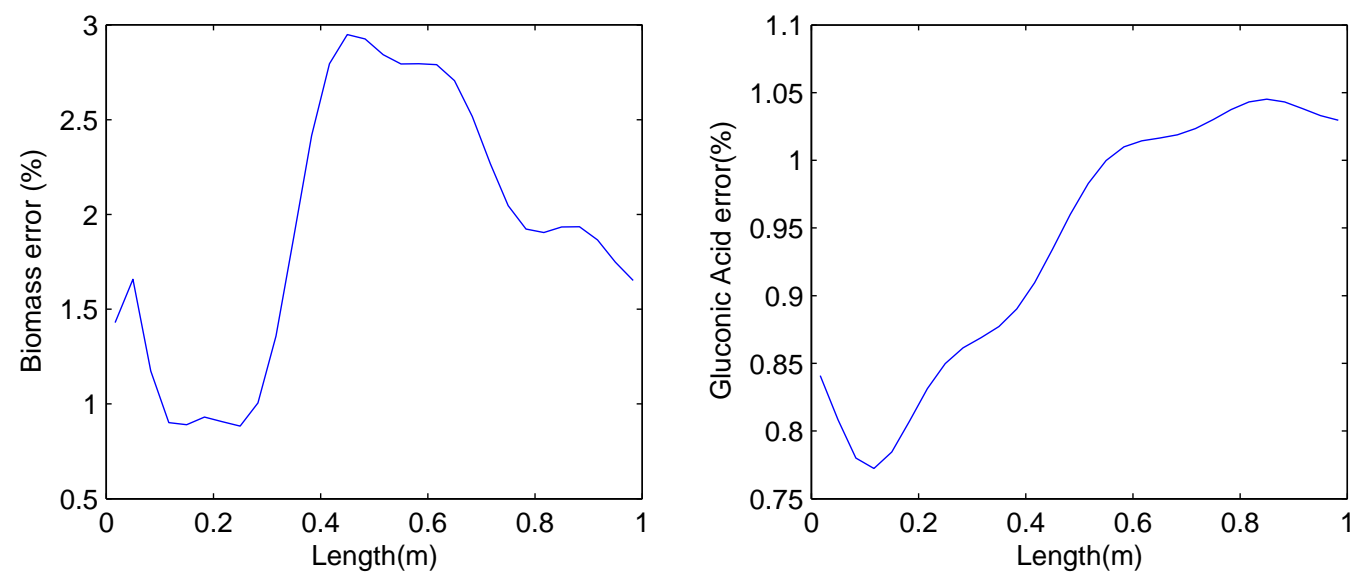

Fig. 9. Distribution of the ROM observation error in the estimation of gluconic acid and biomass from partial measurements at final time 


\section{List of Tables}

1 Algebraic relations to numerically compute integrals and derivatives using the FEM structure. 


\begin{tabular}{|c|c|c|}
\hline Continous & & Discrete \\
\hline$\langle\mathrm{g}(\xi), \mathrm{f}(\xi)\rangle_{\Omega}$ & $\longrightarrow$ & $\mathrm{G}^{\mathrm{T}}(\mathcal{D} \mathcal{A}) \mathrm{F}$ \\
\hline$\left\langle\mathrm{g}(\xi), \frac{\partial \mathrm{f}(\xi)}{\partial \xi}\right\rangle_{\Omega}$ & $\longrightarrow$ & $\mathrm{G}^{\mathrm{T}}(\mathcal{B E}) \mathrm{F}$ \\
\hline$\left\langle\mathrm{g}(\xi), \frac{\partial^{2} \mathrm{f}(\xi)}{\partial \xi^{2}}\right\rangle_{\Omega}$ & $\longrightarrow$ & $\mathrm{G}^{\mathrm{T}}(\mathcal{G})-\mathrm{G}^{\mathrm{T}}(\mathcal{C}+v \mathcal{Q}) \mathrm{F}$ \\
\hline$\frac{\partial \mathrm{f}(\xi)}{\partial \xi}$ & $\longrightarrow$ & $\left(\mathcal{D} \mathcal{A}^{-1}\right)(\mathcal{B E}) \mathrm{F}$ \\
\hline$D \frac{\partial^{2} \mathrm{f}(\xi)}{\partial \xi^{2}}$ & $\longrightarrow$ & $\left.\mathcal{A}^{-1}\right)(\mathcal{G})-\left(\mathcal{D} \mathcal{A}^{-1}\right)(D \mathcal{C}+\mathcal{Q}) \mathrm{F}$ \\
\hline
\end{tabular}

Table 1

Algebraic relations to numerically compute integrals and derivatives using the FEM structure. 


\begin{tabular}{|c||c|}
\hline Design parameter & Symbol Value \& Units \\
\hline Mass dispersion coefficient & $\mathrm{D}=0.01 \mathrm{~m}^{2} \mathrm{~h}^{-1}$ \\
\hline Flow velocity & $\mathrm{v}=0.01 \mathrm{mh}^{-1}$ \\
\hline Mass exchange parameter & $\mathrm{kla}=600 \mathrm{~h}^{-1}$ \\
\hline \hline Input related parameters & \\
\hline Saturation of dissolved oxygen & $O_{2}^{*}=7.5 \mathrm{e}-3 \mathrm{gl}^{-1}$ \\
\hline Glucose stream & $G^{\text {in }}=115 \mathrm{gl}^{-1}$ \\
\hline \hline Dissolved oxygen stream & $O_{2 i n}=7.5 \mathrm{e}-3 \mathrm{gl}^{-1}$ \\
\hline $\begin{array}{c}\text { Biomass reaction } \\
\text { rate parameters }\end{array}$ & Gluconic acid reaction \\
\hline$\mu_{\text {max }}^{X}=0.219 \mathrm{~h}^{-1}$ & $\mu_{\text {max }}^{G A}=0.312 \mathrm{~h}^{-1}$ \\
\hline$k_{1}^{X}=2.53 \mathrm{gl}^{-1}$ & $k_{1}^{G A}=109 \mathrm{gl}^{-1}$ \\
\hline$k_{2}^{X}=5 \mathrm{gl}^{-1}$ & $k_{2}^{G A}=6 \mathrm{gl}^{-1}$ \\
\hline
\end{tabular}

Table 2

Model Parameters 


\section{Notation}

\begin{tabular}{|c|c|c|}
\hline$A_{o}$ & Matrix used to obtain the independent reaction rate fields & $\mathbb{R}^{s-r \times s}$ \\
\hline$B$ & Basis for the null space of $K^{\mathrm{T}}$ & $\mathbb{R}^{s \times s-r}$ \\
\hline $\mathcal{B E}$ & FEM matrix associated with the gradiant operator & $\mathbb{R}^{n \times n}$ \\
\hline$c^{a}$ & Modes or time-dependent functions associated with $a$ & $\mathbb{R}$ \\
\hline$C^{a}$ & Vector collecting the set $\left\{c_{i}^{a}\right\}_{i=1}^{m_{a}}$ & $\mathbb{R}^{m_{a}}$ \\
\hline $\mathcal{C}$ & FEM matrix associated with the laplacian operator & $\mathbb{R}^{n \times n}$ \\
\hline$d_{k}$ & Diffusion associated with the kth field of $x_{e}$ & $\mathbb{R}^{+}$ \\
\hline$D$ & Matrix of dispersion parameters & $\mathbb{R}^{s \times s}$ \\
\hline $\mathcal{D} \mathcal{A}$ & FEM matrix associated with the integral operator & $\mathbb{R}^{n \times n}$ \\
\hline$e_{k}$ & Error between the "real" and the kth observed field of $x_{e}$ & $\mathbb{R}$ \\
\hline$e_{p k}$ & Error between the "real" and the observed $p_{k}$ & $\mathbb{R}$ \\
\hline $\mathcal{G}$ & FEM vector associated with the boundary conditions & $\mathbb{R}^{n}$ \\
\hline$h$ & Dimension of the spatial domain & $\mathbb{N}$ \\
\hline$K$ & Matrix of yield coefficients & $\mathbb{R}^{s \times r}$ \\
\hline$l$ & Number of uncorrelated snapshots & $\mathbb{N}$ \\
\hline$L$ & Axial dimension of the reactor & $\mathbb{R}^{+}$ \\
\hline$n$ & Dimension of the FEM mesh & $\mathbb{N}$ \\
\hline$N$ & Number of non-overlapping subdomains so $\Omega=\cup_{j=1}^{N} \Omega_{j}$ & $\mathbb{N}$ \\
\hline$N_{s}$ & Number of sampling times & $\mathbb{R}$ \\
\hline$p$ & $\begin{array}{l}\text { Independent reaction rates field with homogeneous bound- } \\
\text { ary conditions }\end{array}$ & $\mathbb{R}^{s-r}$ \\
\hline$P_{k}$ & Spatial discretization of $p$ in the FEM mesh & $\mathbb{R}^{1 \times n}$ \\
\hline$q_{k}$ & $\begin{array}{l}\text { Transfer coefficient with environment associated with the } \\
\text { kth field of } x_{e}\end{array}$ & {$[0, \infty)$} \\
\hline$Q$ & Matrix of exchange coefficients with the environment & $\mathbb{R}^{s \times s}$ \\
\hline $\mathcal{Q}$ & FEM vector associated with the boundary conditions & $\mathbb{R}^{n}$ \\
\hline$r$ & Dimension of the reaction rates vector & $\mathbb{N}$ \\
\hline$R$ & Kernel of both LSD and POD method & $\mathbb{R}$ \\
\hline $\mathcal{R}$ & Spatial discretization of $R$ in the FEM mesh & $\mathbb{R}^{n \times n}$ \\
\hline$s$ & Number of states & $\mathbb{N}$ \\
\hline$t$ & Time & {$[0, \infty)$} \\
\hline$v$ & Flow velocity & $\mathbb{R}^{+}$ \\
\hline $\mathcal{V}$ & Lyapunov function & {$[0, \infty)$} \\
\hline$x$ & State vector field & $\mathbb{R}^{s}$ \\
\hline$y$ & Each element of the measurement vector & $\mathbb{R}$ \\
\hline$z$ & Vector field independent of the reaction rates & $\mathbb{R}^{s-r}$ \\
\hline
\end{tabular}


Greek symbols

$\Gamma \quad$ Boundary of the spatial domain

$\mathbb{R}^{h-1}$

$\lambda^{a} \quad$ Eigenvalue associated with the field $a$

$\mathbb{R}$

$\Lambda^{a} \quad$ Diagonal matrix collecting the set $\left\{\lambda_{i}^{a}\right\}_{i=1}^{m_{a}}$

$\mathbb{R}^{m_{a} \times m_{a}}$

$\xi \quad$ Spatial coordinates

$\mathbb{R}^{h}$

$\phi^{a} \quad$ Global basis function associated with the filed a

$\mathbb{R}$

$\psi \quad$ FEM locally basis function $(i=1, \ldots, n)$

$\mathbb{R}$

$\epsilon_{k} \quad$ Truncation error of $p_{k}$

$\mathbb{R}$

$\Omega \quad$ Spatial domain

$\mathbb{R}^{h}$

$\Omega_{\mathcal{S}} \quad$ Spatial domain of measurements

$\mathbb{R}^{n}$

$\varphi \quad$ Vector of reaction rates

$\mathbb{R}^{r}$

Superindex

$a^{i n} \quad a$ input in the reactor

$a^{\mathrm{T}} \quad$ Transpose of $a$

$a^{*} \quad a$ property in the environment

$\bar{a} \quad$ Transformation of the field $a$

$\widetilde{a} \quad$ Truncation of the infinite dimensional field $a$

$\widehat{a} \quad$ Truncation of the infinite dimensional field $a$

Subindex

$a_{e} \quad$ Partition of the matrix or vector $a$ associated with the observed states

$a_{k} \quad$ Element (or row in the case of full matrices) associated with the kth element of $a(k=1, \ldots, s-r)$

$a_{m} \quad$ Partition of the matrix or vector $a$ associated with the measurable states

$a_{\mathcal{S}} \quad a$ field in the $\Omega_{\mathcal{S}}$ spatial domain

$a_{0} \quad$ Initial condition of $a$

Table 3

Nomenclature denoting by $a$ a generic function 


\section{Appendices}

\section{A Optimal field reconstruction}

Let us denote each element of the measurement vector $x_{m}$ as $y$ and expand it in terms of the basis function set $\left\{\phi_{i}^{y}(\xi)\right\}_{i=1}^{m_{y}}$ in the same way as in (22), so that:

$$
y \cong\left[\phi_{1}^{y}, \ldots, \phi_{m_{k}}^{y}\right] C^{y} \equiv \Phi^{y} C^{y}
$$

Discrete global basis functions $\Phi^{y}$ are obtained off-line from the POD method representing the behaviour not only related to the diffusion part, as in the LSD, but also with the reaction rates and the rest of transport terms. To that purpose a sufficiently rich collection of $l$ uncorrelated snapshots of the states are taken at every point of the FEM mesh, denoted by $Y\left(t_{j}\right)$, and used to compute the kernel of the eigenvalue problem associated with (21):

$$
\mathcal{R} \mathcal{D} \mathcal{A} \Phi_{i}^{y}=\lambda_{i}^{y} \Phi_{i}^{y} \quad \text { with } \quad R=\frac{1}{l} \sum_{j=1}^{l}\left[Y\left(t_{j}\right) Y\left(t_{j}\right)^{\mathrm{T}}\right]
$$

Details about the POD implementation exploiting the FEM structure and the continuous formulation can be also seen in the García et al. work ${ }^{12}$.

In the same work, the notion of subdomains of measurements was also introduced as those which partition the whole spatial domain and are suitable for placing sensor arrays. Thus the estimation of the vector field $x_{m}$ from optimal selection of point-wise measurements is substituted by the problem of reconstructing spatial distributed states searching among regions of measurements ensuring the solvability of the problem in high dimensionality FEM meshes. For that purpose let us decompose the spatial domain $\Omega$ into $N$ nonoverlapping subdomains $\Omega_{j}$ so $\Omega=\cup_{j \in \mathcal{J}} \Omega_{j}$ where $\mathcal{J}$ defines the set of natural numbers ordered from 1 to $N$. With these preliminaries, the reconstruction problem can be stated as follows:

For a given set of subdomains $\Omega_{\mathcal{S}}=\cup_{j \in \mathcal{S}} \Omega_{j} \subset \Omega$ with $\mathcal{S} \subset \mathcal{J}$ (i.e. a collection of elements belonging to $\mathcal{J}$ ) where the sensors are located, find the mode vector $C^{y}$ associated with the low-dimensional basis set (defined over $\Omega_{\mathcal{S}}$ ) which minimizes the distance between the measurements $y_{\mathcal{S}}$ and the estimates $\widehat{y}_{\mathcal{S}}$ over the $L^{2}$ norm

Before proceeding with the reconstruction problem, let us first note that basis functions are orthogonal and can be normalized with respect to the spatial integration operator $\mathcal{D} \mathcal{A}$ so that $\Phi^{y \mathrm{~T}} \mathcal{D} \mathcal{A} \Phi^{y}=I$, with $I$ denoting the identity matrix. In the same way, the above orthonormality condition can be re-written 
as:

$$
\sum_{i=1}^{N} \Phi_{i}^{y \mathrm{~T}} \mathcal{D} \mathcal{A}_{i} \Phi_{i}^{y}=I
$$

where $\Phi_{i}^{y} \in \mathbb{R}^{n_{i} \times m_{k}}, \mathcal{D} \mathcal{A}_{i} \in \mathbb{R}^{n_{i} \times n_{i}}$ and $n_{i}$ are defined for each subdomain. In particular, $\mathcal{D} \mathcal{A}_{i}$ corresponds with the spatial integration operator associated to subdomain $i$ which, as $\mathcal{D} \mathcal{A}$, is invertible and thus accepts the following factorization:

$$
\mathcal{D} \mathcal{A}_{i}=W_{i} \Lambda_{i} W_{i}^{\mathrm{T}}
$$

with $\Lambda_{i}$ being diagonal and $W_{i}$ unitary matrices.

Using the Table 1 and expressing the discrete version of the estimates as in (A.1), the optimization problem is formally stated as:

$$
\min _{\widehat{C}^{y}} \frac{1}{2}\left\|e^{y}\right\|_{\Omega_{\mathcal{S}}}^{2}=\min _{\widehat{C}^{y}} \sum_{i \in \mathcal{S}}\left[\left(y_{i}-\widehat{C}^{y} \Phi_{i}^{y}\right)^{\mathrm{T}} \mathcal{D} \mathcal{A}_{i}\left(y_{i}-\widehat{C}^{y} \Phi_{i}^{y}\right)\right]
$$

Its solution, once cast into a least squares minimization problem ${ }^{12}$, takes the form:

$$
\widehat{C}^{y}=\left(\Pi^{y}\right)^{-1} \sum_{i \in \mathcal{S}} \Phi_{i}^{y \mathrm{~T}} \mathcal{D} \mathcal{A}_{i} y_{i} \quad \Pi^{y}=\sum_{i \in \mathcal{S}} \mathcal{Z}_{i}^{y \mathrm{~T}} \mathcal{Z}_{i}^{y}
$$

where matrices $\mathcal{Z}_{i}^{y}$ are computed as:

$$
\mathcal{Z}_{i}=\sqrt{\Lambda_{i}^{y}} W_{i}^{\mathrm{T}} \Phi_{i}^{y} \quad \text { with } \quad \Phi_{i}^{y \mathrm{~T}} \mathcal{D} \mathcal{A}_{i} \Phi_{i}^{y}=\mathcal{Z}_{i}^{y \mathrm{~T}} \mathcal{Z}_{i}^{y}
$$

It must be pointed out that matrix $\Pi$ is always invertible provided that the number of point-wise measurements is larger than or equal to the dimension of the reduced space. Under this assumption the vector $\widehat{C}^{y}$ is identifiable and the errors $e^{y}$ bounded. The same assumption was also made by Christofides and Baker ${ }^{31}$ to deal with time-varying uncertain variables in designing output feedback controllers. According with this work, although the static output feedback is more sensitive to measurements noise, the boundedness of the state and output tracking was guaranteed provided that the separation between the slow and fast eigenvalues is sufficiently large.

Equation (A.6) suggests a criterion to place sensors based on the degree of conditioning of matrix $\Pi$. In this way, sensors will be placed over those subdomains $\mathcal{S}$ which maximize the minimum eigenvalue of $\Pi$. Formally this problem is stated as:

$$
\max _{\mathcal{S}} \min _{j} \lambda_{j}^{y}\left(\sum_{i \in \mathcal{S}} \mathcal{Z}_{i}^{y \mathrm{~T}} \mathcal{Z}_{i}^{y}\right)
$$

and solved by means of the guided search algorithm developed by Alonso et al. ${ }^{7}$. 


\section{B Proof of Proposition 2}

Proof: Combining equations (37) and (38) the observation error then becomes of the form:

$$
e_{k}=z_{k}\left(x_{m}\right)-\widetilde{p}_{k}\left(\widehat{x}_{m}\right)+\left.\frac{1}{v} g_{k}\left(\widehat{x}_{m}, x^{i n}\right)\right|_{\xi=0}-A_{o k}\left(x_{m}-\widehat{x}_{m}\right)
$$

furthermore, using (23) and (36), this error can be re-written as:

$$
e_{k}=p_{k}\left(x_{m}\right)-\widetilde{p}_{k}\left(\widehat{x}_{m}\right)+\left.\frac{1}{v} g_{k}\left(e_{m}\right)\right|_{\xi=0}-A_{o k} e_{m}
$$

with

$$
g_{k}\left(e_{m}\right)=\left.g_{k}\left(x_{m}, x^{i n}\right)\right|_{\xi=0}-\left.g_{k}\left(\widehat{x}_{m}, x^{i n}\right)\right|_{\xi=0}=v\left(d_{k} A_{o k} D_{m}^{-1}-A_{o k}\right) e_{m}
$$

Finally, adding and subtracting $\widehat{p}_{k}\left(\widehat{x}_{m}\right)$ and using equation (34) the observation error can be formally stated as a function of the truncation error $\epsilon_{k}$, and the $p_{k}$ error, defined as $e_{p k}=p_{k}\left(x_{m}\right)-\widehat{p}_{m}\left(\widehat{x}_{m}\right)$ :

$$
e_{k}=e_{p k}+\left.\frac{1}{v} g_{k}\left(e_{m}\right)\right|_{\xi=0}-A_{o k} e_{m}+\epsilon_{k}
$$

Before continuing, let us study the stability properties of $e_{p k}$ whose evolution can be obtained by subtracting equation (25) from (26) where the reconstruction of the measurable fields $\widehat{x}_{m}$ has been employed:

$$
\begin{gathered}
\frac{\partial e_{p k}}{\partial t}=d_{k} \frac{\partial^{2} e_{p k}}{\partial \xi^{2}}-v \frac{\partial e_{p k}}{\partial \xi}-q_{k} e_{p k}+f_{k}\left(e_{m}\right) \\
d_{k} \frac{\partial e_{p k}(0, t)}{\partial \xi}=v e_{p k}(0, t), \quad \frac{\partial e_{p k}(L, t)}{\partial \xi}=0 \\
e_{p k}(\xi, 0)=e_{p k}^{0}
\end{gathered}
$$

where

$$
\begin{aligned}
f_{k}\left(e_{m}\right) & =f_{k}\left(x_{m}, x^{*}, x^{i n}\right)-f_{k}\left(\widehat{x}_{m}, x^{*}, x^{i n}\right)= \\
& =\left.\frac{q_{k}}{v} g_{k}\left(e_{m}\right)\right|_{\xi=0}+h_{k}\left(e_{m}\right)+\left.\frac{1}{v} \dot{g}_{k}\left(e_{m}\right)\right|_{\xi=0} \\
h_{k}\left(e_{m}\right) & =h_{k}\left(x_{m}, x^{*}\right)-h_{k}\left(\widehat{x}_{m}, x^{*}\right) \\
& =\left(A_{o k} D_{m}-d_{k} A_{o k}\right) \frac{\partial^{2} e_{m}}{\partial \xi^{2}}-\left(A_{o k} Q_{m}-q_{k} A_{o k}\right) e_{m}
\end{aligned}
$$

For the sake of clarity, let us omit the subindex $k$ and follow the next steps: 
(1) Make use of Lemma 1, analogously to proposition 1, and re-write the error $e_{p k}$ as:

$$
e_{p}=\exp (\mu \xi-\delta t) \bar{e} \quad \delta=q+\frac{v^{2}}{4 d}, \quad \mu=\frac{v}{2 d}
$$

where the dynamics of the $\bar{e}$ field reads:

$$
\begin{gathered}
\frac{\partial \bar{e}}{\partial t}=d \frac{\partial^{2} \bar{e}}{\partial \xi^{2}}+\exp (\delta t-\mu \xi) f\left(e_{m}\right) \\
\frac{\partial \bar{e}(0, t)}{\partial \xi}=\mu \bar{e}(0, t) \quad \frac{\partial \bar{e}(L, t)}{\partial \xi}=-\mu \bar{e}(L, t) \\
\bar{e}(\xi, 0)=\bar{e}^{0}=e_{p}^{0} \exp (-\mu \xi)
\end{gathered}
$$

(2) Define a Lyapunov function $\mathcal{V}=\frac{1}{2}\langle\bar{e}, \bar{e}\rangle_{\Omega}$ and compute its time derivative along the trajectories (B.4a):

$$
\frac{\partial \mathcal{V}}{\partial t}=d\left\langle\bar{e}, \frac{\partial^{2} \bar{e}}{\partial \xi^{2}}\right\rangle_{\Omega}+\left\langle\bar{e}, \exp (\delta t-\mu \xi) f\left(e_{m}\right)\right\rangle_{\Omega}
$$

(3) Find bounds for the right hand side in terms of $\|\bar{e}\|_{\Omega}$. We have already found in the demonstration of proposition 1 that $d\left\langle\bar{e}, \frac{\partial^{2} \bar{e}}{\partial \xi^{2}}\right\rangle_{\Omega} \leq-d \nu\|\bar{e}\|_{\Omega}^{2}$. In order to bound the second right hand term note that, since $e_{m}$ and its second spatial derivative $\frac{\partial^{2} e_{m}}{\partial e_{m}^{2}}$ are bounded, we can consider a parameter $F$ so that $F \geq f\left(e_{m}\right)$ and employ the Schwarz inequality to state:

$$
\left\langle\bar{e}, \exp (\delta t-\mu \xi) f\left(e_{m}\right)\right\rangle_{\Omega} \leq \kappa F \exp (\delta t)\|\bar{e}\|_{\Omega}, \quad \kappa=\left[\frac{1-\exp (-2 \mu L)}{2 \mu}\right]^{1 / 2}
$$

(4) Re-write equation (B.5) noting that $\mathcal{V}=\frac{1}{2}\|\bar{e}\|_{\Omega}^{2}$ as follows:

$$
\dot{\mathcal{V}} \leq-2 d \nu \mathcal{V}+\kappa F \exp (\delta t) \sqrt{2 \mathcal{V}}
$$

(5) Use the transformation $\mathscr{V}=\sqrt{\mathcal{V}}$ and differentiate $\mathscr{V}$ to obtain:

$$
\dot{\mathscr{V}}+d \nu \mathscr{V} \leq \frac{\kappa F}{\sqrt{2}} \exp (\delta t)
$$

(6) Multiply both sides by $\exp (d \nu t)$, use the transformation $r=\mathscr{V} \exp (d \nu t)$ so that

$$
\dot{r} \leq \frac{\kappa F}{\sqrt{2}} \exp [(d \nu+\delta) t]
$$

and integrate the inequality to get:

$$
\mathscr{V} \exp (d \nu t)-\mathscr{V}^{0} \leq \frac{\kappa F}{\sqrt{2}(d \nu+\delta)} \exp [(d \nu+\delta) t]-\frac{\kappa F}{\sqrt{2}(d \nu+\delta)}
$$


(7) Since $\mathscr{V}=\frac{1}{\sqrt{2}}\|\bar{e}\|_{\Omega}$, we then have

$$
\|\bar{e}\|_{\Omega} \leq \frac{\kappa F}{d \nu+\delta} \exp (\delta t)+\left[\left\|\bar{e}^{0}\right\|_{\Omega}-\frac{\kappa F}{d \nu+\delta}\right] \exp (-d \nu t)
$$

Finally, let us re-introduce the subindex $k$ and combine relation (B.3) with (B.1) to get:

$$
\left\|e_{k}\right\|_{\Omega} \leq\left\|\exp \left(\mu_{k} \xi-\delta_{k} t\right)\right\|_{\Omega}\left\|\bar{e}_{k}\right\|_{\Omega}+\left\|\left.\frac{1}{v} g_{k}\left(e_{m}\right)\right|_{\xi=0}+\epsilon_{k}-A_{o k} e_{m}\right\|_{\Omega},
$$

therefore the norm of the observation error, using inequality (B.6) and rearranging terms, is bounded as:

$$
\left\|e_{k}\right\|_{\Omega} \leq \varpi_{k} \exp \left[-\left(\delta_{k}+d_{k} \nu_{k}\right) t\right]+\Xi_{k}
$$

with

$$
\begin{aligned}
\varpi_{k} & =\left\|\bar{e}_{k}^{0}\right\|_{\Omega}\left[\frac{\exp \left(2 \mu_{k} L\right)-1}{2 \mu_{k}}\right]^{1 / 2}-\eta_{k}<\infty \\
\Xi_{k} & =\eta_{k}+\left\|\left.\frac{1}{v} g_{k}\left(e_{m}\right)\right|_{\xi=0}+\epsilon_{k}-A_{o k} e_{m}\right\|_{\Omega}<\infty \\
\eta_{k} & =\frac{F_{k}}{d_{k} \nu_{k}+\delta_{k}}\left[\frac{\exp \left(2 \mu_{k} L\right)+\exp \left(-2 \mu_{k} L\right)-2}{4 \mu_{k}^{2}}\right]^{1 / 2}
\end{aligned}
$$

\section{References}

[1] Bastin G, Dochain D. On-line estimation and adaptive control bioreactors. Amsterdam: Elsevier, 1990.

[2] Dochain D, Perrier M, Ydstie BE. Asymptotic observers for stirred tank reactors. Chemical Engineering Science. 1992;47:4167-4177.

[3] Dochain D, Tali-Maamar N, Babary JP. On modelling, monitoring and control of fixed bed bioreactors. Computers chem. Engng. 1996;21:12551266.

[4] Dochain D. State Observers for Tubular Reactors with Unknown Kinetics. Journal of process control. 2000;10:259-268.

[5] Christofides PD. Nonlinear and Robust Control of PDE Systems: Methods and Applications to Transport-Reaction Processes. Boston: Birkhäuser, 2001.

[6] Vande Wouwer A, Point N, Remy R. An approach to the selection of optimal sensor locations in distributed parameter systems. Journal of Process Control. 2000;10:291-300. 
[7] Alonso A.A, Frouzakis CE, Kevrekidis IG. Optimal Sensor Placement for State Reconstruction of Distributed Process Systems. AIChE Journal. 2004;50:1438-1452.

[8] Alonso AA, Fernández CV, Banga JR. Dissipative Systems: from physics to robust nonlinear control. Int. J. Robust Nonlinear Control. 2004;14:157-179.

[9] Balsa-Canto E, Alonso AA, Banga JR. Reduced-order models for nonlinear distributed process systems and their application in dynamic optimization. Industrial \& Engineering Chemistry Research. 2004;43:33533363.

[10] Alonso AA, Banga JR, Sánchez I. Passive Control Design for Distributed Process Systems: Theory and Applications. AIChE Journal. 2000;46:1593-1606.

[11] Alonso AA, Ydstie BE. Stabilization of Distributed Systems Using Irreversible Thermodynamics. Automatica. 2001;37:1739-1755.

[12] García MR, Vilas C, Banga JR, Alonso AA. Optimal Field Reconstruction of Distributed Process Systems from Partial Measurements. Industrial \& Engineering Chemistry Research. 2007;46:530-539.

[13] Reddy JN. An Introduction to the Finite Element Method (2nd edition). United States: McGraw-Hill, 1993.

[14] Dochain D. State and parameter estimation in chemical and biochemical processes: a tutorial. Journal of Process Control. 2003;13:801-818.

[15] Winkin JJ, Dochain D, Ligarius P. Dynamical analysis of distributed parameter tubular reactors. Automatica. 1992;36:349-361.

[16] Polyanin AD. Handbook of Linear Partial Differential Equations for Engineers and Scientist. Boca Raton: Chapman \& Hall, 2002.

[17] Morrey C. Multiple Integrals in the Calculus of Variations. New York:Springer-Verlag, 1966.

[18] Smoller J. Shock Waves and Reaction-Diffusion Equations (2nd edition). New york: Springer-Verlag, 1994.

[19] Alonso AA, Kevrekidis IG, Banga JR, Frouzakis CE. Optimal sensor location and reduced order observer design for distributed process systems. Computers and Chemical Engineering. 2004;28:27-35.

[20] Delattre C, Dochain D, Winkin J. Observability analysis of nonlinear tubular (bio)reactor models: a case study. Journal of Process Control. 2004;14:661-669.

[21] Christofides PD, Daoutidis P. Finite-Dimensional Control of Parabolic PDE systems using approximate inertial manifolds. J. Math. Anal Appl.. 1997;216:398-420.

[22] Baker J, Christofides PD. Finite Dimensional Approximation and Control of Nonlinear Parabolic PDE. Systems Internat. J. Control. 2000;73:439456.

[23] Balsa-Canto E, Alonso AA, Banga JR. A novel, efficient and reliable method for thermal process design and optimization. Part I: Theory. Journal of Food Engineering. 2002;52:227-234. 
[24] Gottlieb D, Orszag SA. Numerical analysis of spectral methods: Theory and applications. Philadelphia: Society for Industrial and Applied Mathematics, Pennsylvania 1977.

[25] Fattorini HO. Boundary Control Systems. SIAM J. Control. 1968;6:349385.

[26] Emirsjlow Z, Townley S. From PDEs with Boundary Control to the Abstract State Equation with an Unbounded Input Operator: A Tutorial. European Journal of Control. 2000;6:27-49.

[27] Courant R, Hilbert D. Methods of Mathematical Physics. New York: John Wiley \& Sons, 1989.

[28] Kubrusly CS, Malebranche H. Sensors and Controllers Location in Distributed Systems-A Survey Automatica. 1992;21:117-128.

[29] Antoniades C, Christofides PD. Integrating nonlinear output feedback control and optimal actuator/sensor placement for transport-reaction processes Chemical Engineering Science. 2001;56:4517-4535.

[30] Mirón J, González MP, Pastrana L, Murado MA. Diauxic Production of Glucose Oxidase by Aspergillus Niger in Submerged Culture. A Dynamic Model Enzime and Microbial Technology. 2002;31:615-620.

[31] Christofides PD, Baker J. Robust output feedback control of quasi-linear parabolic PDE systems. Syst. \& Cont. Letters. 1999;36:307-316. 